Astronomy and Astrophysics Review Manuscript-Nr.

(will be inserted by hand later)

\title{
Gravitational Lensing in Quasar Samples
}

\author{
Jean-François Claeskens` and Jean Surdej ${ }^{\star \star}$ \\ Institut d'Astrophysique et de Géophysique, Université de Liège, Avenue de Cointe 5, B-4000 \\ Liège, Belgium
}

Received: 4 April 2000 / Accepted:

Summary. The first cosmic mirage was discovered approximately 20 years ago as the double optical counterpart of a radio source. This phenomenon had been predicted some 70 years earlier as a consequence of General Relativity. We present here a summary of what we have learnt since. The applications are so numerous that we had to concentrate on a few selected aspects of this new field of research.

This review is focused on strong gravitational lensing, i.e. the formation of multiple images, in QSO samples. It is intended to give an up-to-date status of the observations and to present an overview of its most interesting potential applications in cosmology and astrophysics, as well as numerous important results achieved so far.

The first Section follows an intuitive approach to the basics of gravitational lensing and is developed in view of our interest in multiply imaged quasars. The astrophysical and cosmological applications of gravitational lensing are outlined in Section 2 and the most important results are presented in Section 5. Sections 3 and 4 are devoted to the observations. Finally, conclusions are summarized in the last Section.

We have tried to avoid duplication with existing (and excellent) introductions to the field of gravitational lensing. For this reason, we did not concentrate on the individual properties of specific lens models, as these are already well presented in Narayan and Bartelmann (1996) and on a more intuitive ground in Refsdal and Surdej (1994). Wambsganss (1998) proposes a broad view on gravitational lensing in astronomy; the reviews by Fort and Mellier (1994) and Hattori et al. (1999) deal with lensing by galaxy clusters; microlensing in the Galaxy and the local group is reviewed by Paczyński (1996) and a general panorama on weak lensing is given by Bartelmann and Schneider (1999). The monograph on the theory of gravitational lensing by Schneider, Ehlers and Falco (1992) also remains a reference in the field.

Key words: Gravitational Lensing (12.07.1) - Cosmology: observations (12.03.3) - Quasars: general (11.17.3) - Galaxies: general (11.07.1)

\footnotetext{
* Chargé de Recherches du F.N.R.S. (Belgium)

$\star \star$ Directeur de Recherches du F.N.R.S. (Belgium)

Correspondence to: surdej@astro.ulg.ac.be
} 


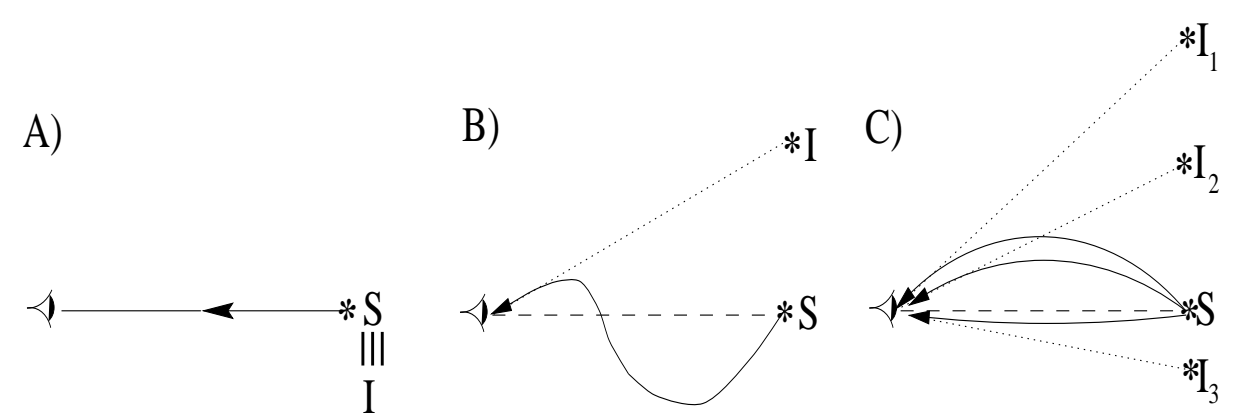

Fig. 1. Light propagation in an euclidian space: A) following a straight line (normal situation); B) following a curved trajectory - in this case, the image position $I$ is different from the source position $S$ as seen by the observer; C) following multiple curved trajectories with the formation of multiple images of the source $S$.

\section{Gravitational lensing}

In this Section, we first propose a general definition of a (cosmic) mirage. We then introduce the basic formalism of the lensing equation, the deflection angle, the amplification and the time delays. Finally, we explain how to find cosmic mirages as efficiently as possible.

\subsection{What is a cosmic mirage?}

People sometimes believe that mirages - alike atmospheric mirages - are synonymous of hallucinations. This is absolutely not the case! Common sense of reality is based on our every-day perception of classical spacetime, whose geometry is flat and static, and where light propagates along straight lines. In this case, we can only see one image of a given point source and it is precisely located in the direction of the source position (see Fig. 1A). The apparent source size (i.e. its solid angle) is then inversely proportional to the square of the source distance. A very general definition of a mirage would be: a distorted view of the reality compared with what is expected by common sense. Following that definition, a mirage may arise in two situations: 1) if the light trajectory is not straight, then the observer sees the image in a direction different from that of the source (Fig. 1B); multiple imaging occurs if more than one light trajectory can reach the observer (Fig. 1C); 2) if space is expanding, as in standard cosmologies, the objects located in the past light-cone of the observer look bigger (or closer) than they actually are at the time of observation (see appearance of sizes in Fig. 2). This "cosmological mirage" is corrected for by the use of cosmological angular distances, which also take into account the effects due to the curvature of the Universe (see Section 1.2).

In this review, we are mainly interested in the first kind of mirages, i.e. when the light trajectories are curved. This happens when the light speed is not isotropic along the directions perpendicular to the light path. For example, since the light speed in a material medium is $v=c / n$ (where $n$ is the refractive index of that medium and $c$ is the light speed in the vacuum), the light "rays" are bent when they travel into an inhomogeneous medium (see Fig. 3). This happens in 


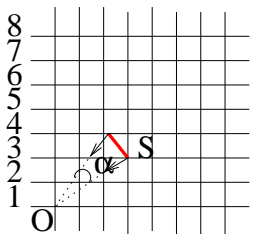

12345678

A) $\mathrm{t}=\mathrm{t}_{\mathrm{em}}$

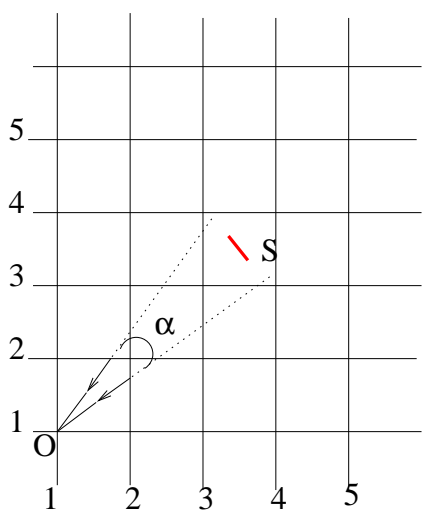

B) $\mathrm{t}=\mathrm{t}_{\mathrm{obs}}$

Fig. 2. Effect due to the expansion of an euclidian space during light travel on the apparent size of distant objects (see text).
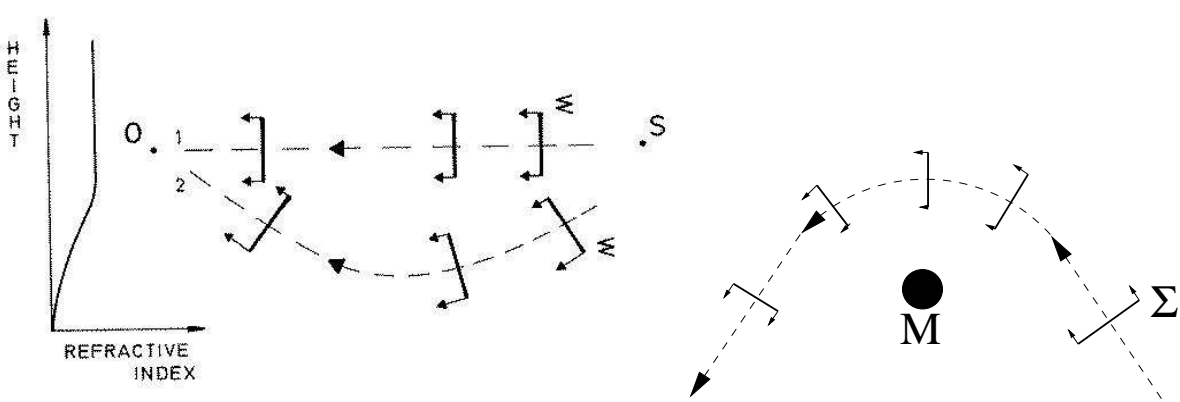

Fig. 3. Left: Deflection of light in an inhomogeneous medium; right: euclidian representation of the gravitational deflection of light. $\Sigma$ refers to a portion of the wavefront. The length of the arrows at the edge of the $\Sigma$ s is proportional to the apparent velocity of light.

the lower atmospheric air layers with strong temperature or density gradients and atmospheric mirages may then be formed (for a more detailed analysis of atmospheric mirages, see Refsdal and Surdej 1994).

As a consequence of General Relativity, spacetime is curved by the gravitational potential $\phi$ associated with a massive object. In the weak field approximation $\left(|\phi| / c^{2} \ll 1\right)$, its metric is simply (e.g. Schutz 1985):

$$
d s^{2}=-\left(1+\frac{2 \phi}{c^{2}}\right) c^{2} d t^{2}+\left(1-\frac{2 \phi}{c^{2}}\right)\left(d x^{2}+d y^{2}+d z^{2}\right) .
$$

However, this new spacetime can be regarded as an euclidian one in which the velocity of light is apparently slowed down in the vicinity of the massive object. Indeed, the light path being defined by the null geodesic $\left(d s^{2}=0\right.$ in Eq. (1)), it is easy to derive that $\phi$ formally acts on the light speed as a medium with an effective refractive index $n_{\phi}$, where

$$
n_{\phi}=1-2 \phi / c^{2} .
$$




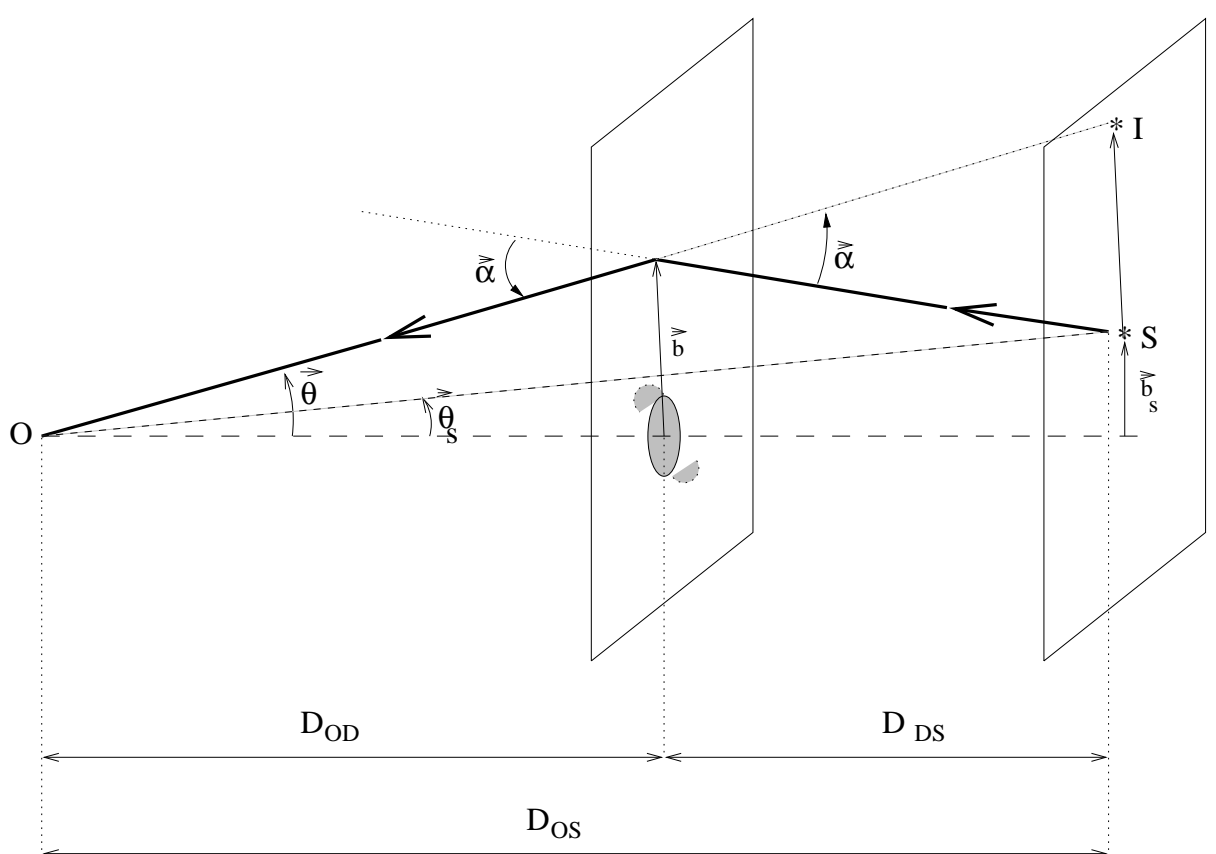

Fig. 4. Gravitational lensing: fundamental scheme.

The gradient of the Newtonian gravitational field $\phi$ in the vicinity of a massive object is now responsible for the angular deflection $\boldsymbol{\alpha}$ of the light trajectories (see Fig. 3). When such a deflected ray reaches the observer, a gravitational mirage is seen and the massive object is called a gravitational lens.

For historical reviews on gravitational lensing, see for example Schneiders, Ehlers and Falco (1992), Refsdal and Surdej (1994) and Wambsganss (1998).

\subsection{The lens equation}

The lens equation connects the source position $\boldsymbol{\theta}_{\mathbf{s}}$ and the position(s) of the image $(\mathrm{s})^{1} \boldsymbol{\theta}$ seen by an observer $O$, given the deflection angle $\boldsymbol{\alpha}$ produced by the gravitational lens. The lens is assumed to be thin and confined into the deflector plane $^{2}$. Fig. 4 shows the geometrical scheme of gravitational lensing. Following rays backward from the observer to the source, it is very easy to derive the lens equation as a relation between the (very small) angles $\boldsymbol{\alpha}, \boldsymbol{\theta}_{\mathbf{s}}$ and $\boldsymbol{\theta}$ :

$$
\boldsymbol{\theta}_{\mathbf{s}}=\boldsymbol{\theta}-\frac{D_{D S}}{D_{O S}} \boldsymbol{\alpha}\left(D_{O D} \boldsymbol{\theta}\right)
$$

\footnotetext{
${ }^{1}$ By analogy with geometrical optics, the observed images are in fact virtual images since they are seen through the lens.

2 The deflection of the light ray is mainly produced around its closest approach to the lens, because astrophysical objects are much smaller than the total length of the light path.
} 
In order to correct for the "cosmological mirage" effect produced by the expansion and the curvature of the Universe, $D_{O D}, D_{O S}$ and $D_{D S}$ must be cosmological angular distances (respectively between the observer and the deflector, the observer and the source and the deflector and the source). In FriedmannLemaître-Robertson-Walker (FLRW) cosmological models with present matter density $\Omega_{o}$ and without cosmological constant $\lambda_{o}$, the angular distance of a source at redshift $z_{S}$ seen from an object at redshift $z_{D}$ is given by

$$
\begin{aligned}
D\left(z_{D}, z_{S}\right)= & \frac{2 c}{H_{o} \Omega_{o}^{2}\left(1+z_{D}\right)\left(1+z_{S}\right)^{2}} \\
& {\left[\left(2+\Omega_{o}\left(z_{S}-1\right)\right) \sqrt{\Omega_{o} z_{D}+1}-\left(2+\Omega_{o}\left(z_{D}-1\right)\right) \sqrt{\Omega_{o} z_{S}+1}\right] . }
\end{aligned}
$$

For other FLRW cosmologies, numerical calculations can be efficiently performed thanks to the ANGSIZ algorithm developed by Kayser et al. (1997).

\subsection{The deflection angle}

The deflection angle $\boldsymbol{\alpha}$ can be estimated in several different ways. A first method is based upon the weak field metric (Eq. (1)) and the geodesic equation (e.g. Weinberg 1972). A second method consists in applying the Fermat Principle to null trajectories in the metric (1) to simultaneously derive the lens equation (3) and the expression of $\boldsymbol{\alpha}$. This physical approach has first been derived by Schneider (1985) and is intuitively presented in Blandford \& Narayan (1986). The deflection angle may also simply be determined from the integration of the variations of the direction $i$ of the ray along the unperturbed trajectory (see Fig. 5 ) by making use of the well known Descartes' law $n \cos i=C^{s t}$ along the light trajectory and assuming small deflection angles $\left(i \ll 1\right.$ since $\phi(b) / c^{2} \ll 1$ for astrophysical objects). Defining $\mathbf{b}$ as the impact parameter in the deflector plane perpendicular to the unperturbed direction of motion $x$ (see Fig. 4):

$$
\mathbf{b}=D_{O D} \boldsymbol{\theta},
$$

we get:

$$
\boldsymbol{\alpha}(\mathbf{b})=-\int_{-\infty}^{+\infty} \frac{d i}{d x} d x=-\int_{-\infty}^{+\infty} \frac{1}{n} \nabla_{\mathbf{b}} n d x=\frac{2}{c^{2}} \int_{-\infty}^{+\infty} \nabla_{\mathbf{b}} \phi d x
$$

where the last equality of Eq. (6) comes from relation (2).

The most basic deflector corresponds to the point mass $M$, whose gravitational potential is simply:

$$
\phi=-\frac{G M}{r}=-\frac{G M}{\sqrt{b^{2}+x^{2}}} .
$$

The deflection angle produced by a point mass is obtained by introducing Eq. (7) into Eq. (6):

$$
\boldsymbol{\alpha}(\mathbf{b})=\frac{4 G M}{c^{2} b^{2}} \mathbf{b} .
$$




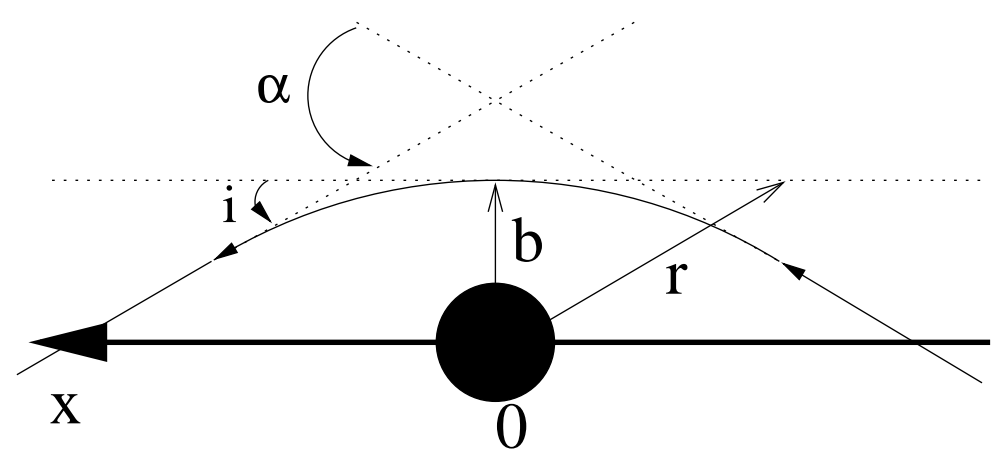

Fig. 5. Deflection of light in the vicinity of a massive object. Descartes' law implies that $\left(1-2 \phi / c^{2}\right) \cos i$ is a constant along the light trajectory. The local variation $d i$ is computed at first order, i.e. along the unperturbed dotted trajectory passing at impact parameter $b(\alpha \ll 1)$.

Under the thin lens approximation, a general deflector is described by its surface mass density $\Sigma(\mathbf{b})$ projected in the deflector plane. Relation (8) for the deflection angle then becomes:

$$
\boldsymbol{\alpha}(\mathbf{b})=\frac{4 G}{c^{2}} \int_{\mathcal{S}} \frac{\Sigma\left(\mathbf{b}^{\prime}\right)\left(\mathbf{b}-\mathbf{b}^{\prime}\right) d b_{1}^{\prime} d b_{2}^{\prime}}{\left|\mathbf{b}-\mathbf{b}^{\prime}\right|^{2}} .
$$

If the deflector is circularly symmetric, relation (9) reduces to:

$$
\boldsymbol{\alpha}(\mathbf{b})=\frac{4 G M(b)}{c^{2} b^{2}} \mathbf{b}
$$

$M(b)$ being the mass inside radius $b$.

Given the expressions (9) or (10) for $\boldsymbol{\alpha}$, the lens equation (3) often has multiple solutions for $\theta$, corresponding to the formation of multiple images of a given source. As an illustration to the case of a point mass deflector $(M(b)=M)$, solving the lens equation yields:

$$
\theta_{1,2}=\frac{1}{2}\left(\theta_{s} \pm \sqrt{\theta_{s}^{2}+4 \theta_{E}^{2}}\right)
$$

\subsection{Two important properties of gravitational lenses}

Let us assume a generic circularly symmetric integrated lens mass profile ${ }^{3}$ :

$$
M(b)=M_{o}\left(\frac{b}{b_{o}}\right)^{\beta} .
$$

\footnotetext{
3 The point mass model, or Schwarzschild lens, is obtained for $\beta=0$ in Eq. (12); $\beta=1$ corresponds to the Singular Isothermal Sphere (SIS); $\beta=2$ yields the uniform sheet of matter (see Narayan and Bartelmann (1996) or Refsdal and Surdej (1994) for general reviews on simple lens models).
} 


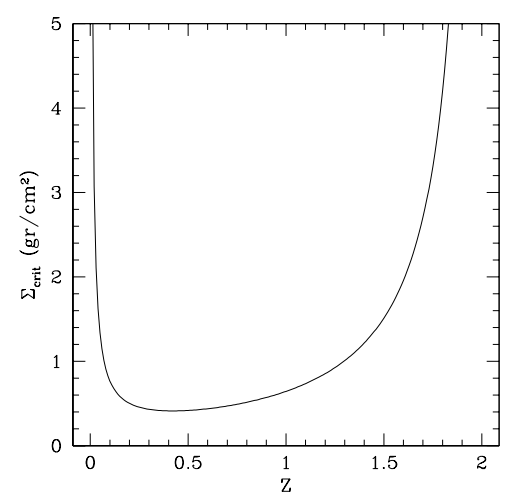

Fig. 6. Critical surface mass density $\Sigma_{\text {crit }}$ as a function of the lens redshift if the source is located at a redshift $z_{s}=2\left(H_{O}=50 \mathrm{~km} / \mathrm{s} / \mathrm{Mpc}\right)$.

In the highly symmetric situation of a perfect alignment between the observer, a circular deflector and a point source, the former sees a ring centered on the deflector. It is called the Einstein ring of the lens (Chwolson 1924, Einstein 1936) and its angular size $\theta_{E}$ satisfies the simplified lens equation: $\theta_{E}=\alpha\left(\theta_{E}\right) \frac{D_{D S}}{D_{O S}}$. From relations (10) and (12), one finds for any value of $\beta<2$ (such that the surface mass density is decreasing with $b$ ):

$$
\theta_{E}=\left(\frac{4 G M_{O}}{c^{2} b_{o}^{\beta}} \frac{D_{D S}}{D_{O D}^{1-\beta} D_{O S}}\right)^{\frac{1}{2-\beta}}=\sqrt{\frac{4 G M_{E}}{c^{2}} \frac{D_{D S}}{D_{O D} D_{O S}}},
$$

where $M_{E}$ is the mass inside the Einstein radius $b_{E}=D_{O D} \theta_{E}$.

Two important properties follow:

1. For small misalignments between the source, the lens and the observer, equation (3) implies that at first order the angular separation between the images $\Delta \theta$ is constant and equal to:

$$
\Delta \theta=2 \theta_{E} .
$$

Thus, from relation (13), the angular separation between the lensed images increases if the lens is more massive or closer to the observer.

2. As a consequence of relation (13), the mean surface mass density within $\theta_{E}$, $\bar{\Sigma}\left(\theta_{E}\right)$, is a constant defined as the critical surface mass density:

$$
\bar{\Sigma}\left(\theta_{E}\right) \equiv \frac{M_{E}}{\pi\left(D_{O D} \theta_{E}\right)^{2}}=\frac{c^{2} D_{O S}}{4 \pi G D_{O D} D_{D S}} \equiv \Sigma_{\text {crit }} .
$$

Relation (15) implies that a finite massive object is a gravitational lens capable to produce multiple images if its central mass density $\Sigma>\Sigma_{\text {crit }}$. The denser the object, the wider the angular separation between the lensed images in order to get the average surface mass density between the images 

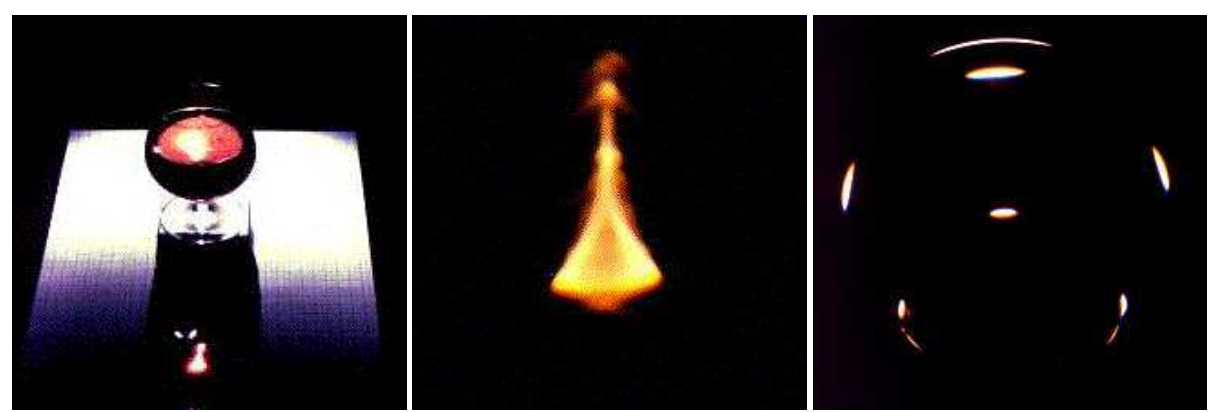

Fig. 7. A glass of wine redistributes the light from a background source. It may form a caustic (left photograph) whose enlargment is shown in the central photograph. Multiple images of the background source are recorded if the objective of a standard camera is located inside the caustic (right photograph).

equal to $\Sigma_{\text {crit }}$. On the contrary, if $\Sigma<\Sigma_{\text {crit }}$ everywhere, the object is not capable to form a cosmic mirage.

Note that $\Sigma_{\text {crit }}$ is only a function of the distances between the source, the lens and the observer. Fig. 6 shows the value of $\Sigma_{\text {crit }}$ as a function of the lens redshift for a distant source located at $z_{s}=2$. A given astrophysical object is a more efficient lens if it is located around $z=0.5$, where $\Sigma_{\text {crit }}$ is minimum and roughly amounts to the surface density of a $1 \mathrm{~cm}$ thick water layer $(\simeq 1$ $\mathrm{g} / \mathrm{cm}^{2}$; see also Section 1.7).

\subsection{Caustics: multiple imaging and amplification}

\subsubsection{Caustics and multiple imaging}

Let us first recall a basic property of optical lenses. A perfectly converging lens focuses the light from a very distant point source located on the optical axis into a single bright point - the focus. Putting one's eye at the focus, one expects to see a deformed and much brighter image of the source. Indeed, the lens does appear uniformly illuminated! If the optical lens suffers from spherical aberrations, its focus is spread along a line segment. An observer located somewhere on this segment then sees a ring of light whose radius depends on his/her distance from the lens; this ring may be compared with the Einstein ring seen through a symmetric gravitational lens.

The optical quality of most natural lenses is very poor; their focus is not a point, nor even a line but a two dimensional envelope called the caustic envelope. The intersection of this envelope with a plane defines a bright closed curve: the caustic line, simply called the caustic. Fig. 7a and b illustrate the caustic created by a glass of wine illuminated by a distant background light source (cf. a candle). An observer putting his/her eye inside the caustic will see multiple images of the source (Fig. 7c). If the observer is not able to resolve the multiple images of the lensed source, he/she will merely detect an increase in the apparent flux coming from the source (see the brighter region of the observer plane enclosed by the caustic in Fig. 7b). 

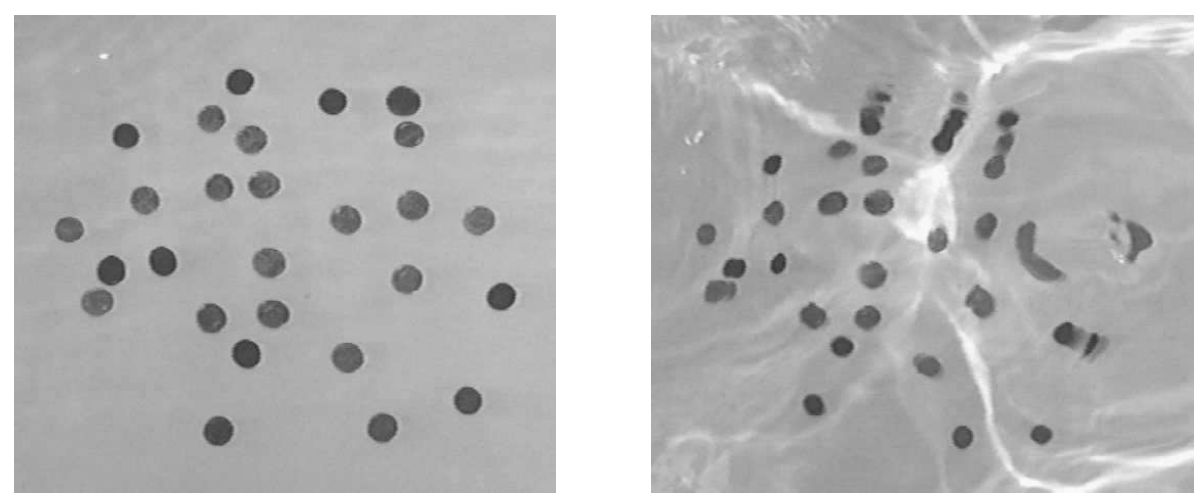

Fig. 8. Another example of caustics. Twenty seven coins have been placed at the bottom of a swimming pool. In the absence of waves, they appear to the observer as seen on the left photograph. At right, the sunlight is refracted through a wavy water surface which creates a complex network of caustics at the bottom of the pool. The wavefronts of sunlight are strongly distorted ... as well as the observed images of the coins. Note that some of the coins (cf. several ones at right) are multiply imaged.

In the formalism of gravitational lensing, the direction "observer - deflector" is used as a reference instead of the direction "source - deflector": the lens equation (3) is therefore a backward ray tracing equation. Caustics in the source plane only differ from those in the observer plane by a scaling factor depending on the distance ratio $D_{O D} / D_{D S}$. Now, for a given observer - deflector direction, a light source is multiply imaged and its apparent luminosity strongly increases if it is located inside the caustic. An example of caustics in the source plane is illustrated in the "swimming pool experiment" (see Fig. 8).

Therefore the area inside the caustic (in the source plane) is a natural cross section associated with multiple imaging; it scales as $\theta_{E}^{2}$. A transition between single imaging and multiple imaging occurs when the source just lies on the caustic: a new single, strongly distorted image is then created.

From a mathematical point of view, the locus of points $\boldsymbol{\theta}$ in the deflector plane where strongly distorted images are formed is defined by those points where the lens equation can not be locally inverted, i.e. where its jacobian $J$ is equal to zero:

$$
J=d t m \mathcal{J}=\left|\frac{\partial \boldsymbol{\theta}_{\mathbf{s}}}{\partial \boldsymbol{\theta}}\right|=0 .
$$

This locus is called the critical line and its mapping onto the source plane by the lens equation yields the caustic.

The shape and number of critical lines and corresponding caustics depend on the exact lens model but for centrally condensed models some generic properties can easily be derived. First, if the lens is circular, Eq. (16) for the critical lines simply reduces to:

$$
J=\frac{\theta_{s} d \theta_{s}}{\theta d \theta}=0 .
$$




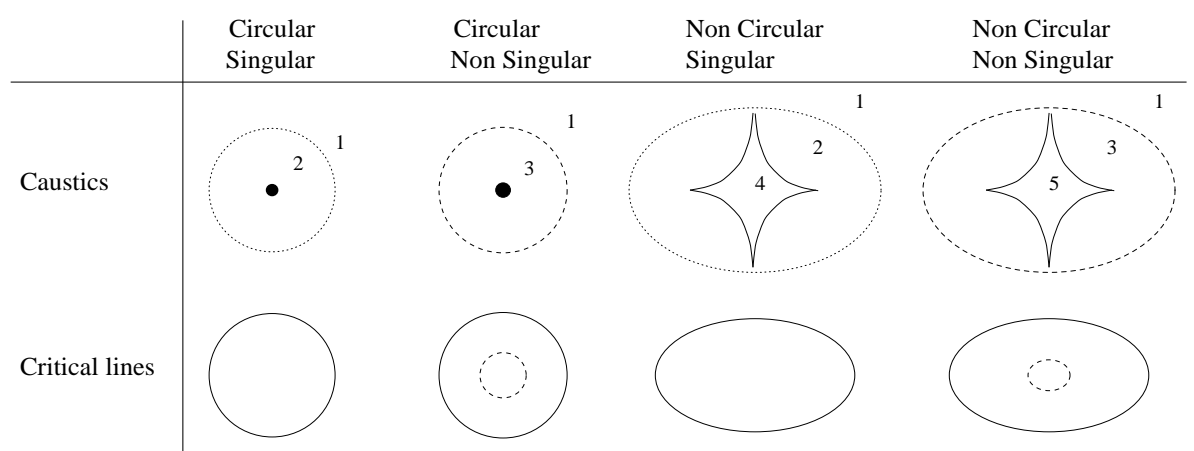

Fig. 9. Number $(1,2,3,4$ or 5) of lensed images, generic shape of caustics and critical lines for (non)singular and (non)circular lens models. The caustic of circular singular models is degenerated into a single point. In the limiting case of an isothermal model, if the source is just inside the "cut" (dotted lines), one secondary image is formed close to the center of the deflector; this "cut" becomes a second caustic (dashed lines) in non singular models and two new images are then produced close to the corresponding critical line. For non circular models, the degeneracy is broken and the inner caustic often has the shape of a diamond; more than 3 images may be produced.

Therefore, in that case there is always one caustic degenerated into the central point $\theta_{s}=0$; the corresponding critical line is the Einstein ring. Then, if the model is non-singular ${ }^{4}$, there is a second circular critical line, called the "radial" critical line and given by the solution of $d \theta_{s} / d \theta=0$ (see Eq. (17)). The caustics produced by circular models are illustrated on the left panels of Fig. 9 .

For a non-circular ${ }^{5}$ model, the degeneracy is broken and the previous inner, point-like caustic often takes the shape of a diamond, allowing the formation of more than 3 images. Asymmetric and non-singular models may form a larger variety of caustics (see e.g. Schneider, Ehlers and Falco 1992, Kassiola and Kovner 1993) but they always produce an odd number of images (Burke 1981). The most common caustics of these models are shown on the right panels of Fig. 9 and representative observed examples are illustrated in Fig. 10.

\footnotetext{
${ }^{4}$ A lens model is singular if $\Sigma(b) \propto b^{-\gamma}$ with $\gamma>0 . \gamma$ must be smaller than 2 to get a finite central mass. It is easy to show from Eq. (10) that in the case of a circular model a radial critical line is only formed if $\gamma<1$. The point mass and the SIS lens models $(\gamma=1)$ are singular models.

5 A non circular model is either a circular model perturbed by an external anisotropy (like a star in the potential of the host-galaxy, Chang \& Refsdal 1979) or an intrinsically asymmetric potential (e.g. Blandford \& Kochanek 1987) or asymmetric mass distribution (e.g. Kassiola \& Kovner 1993, Kormann et al. 1994)
} 

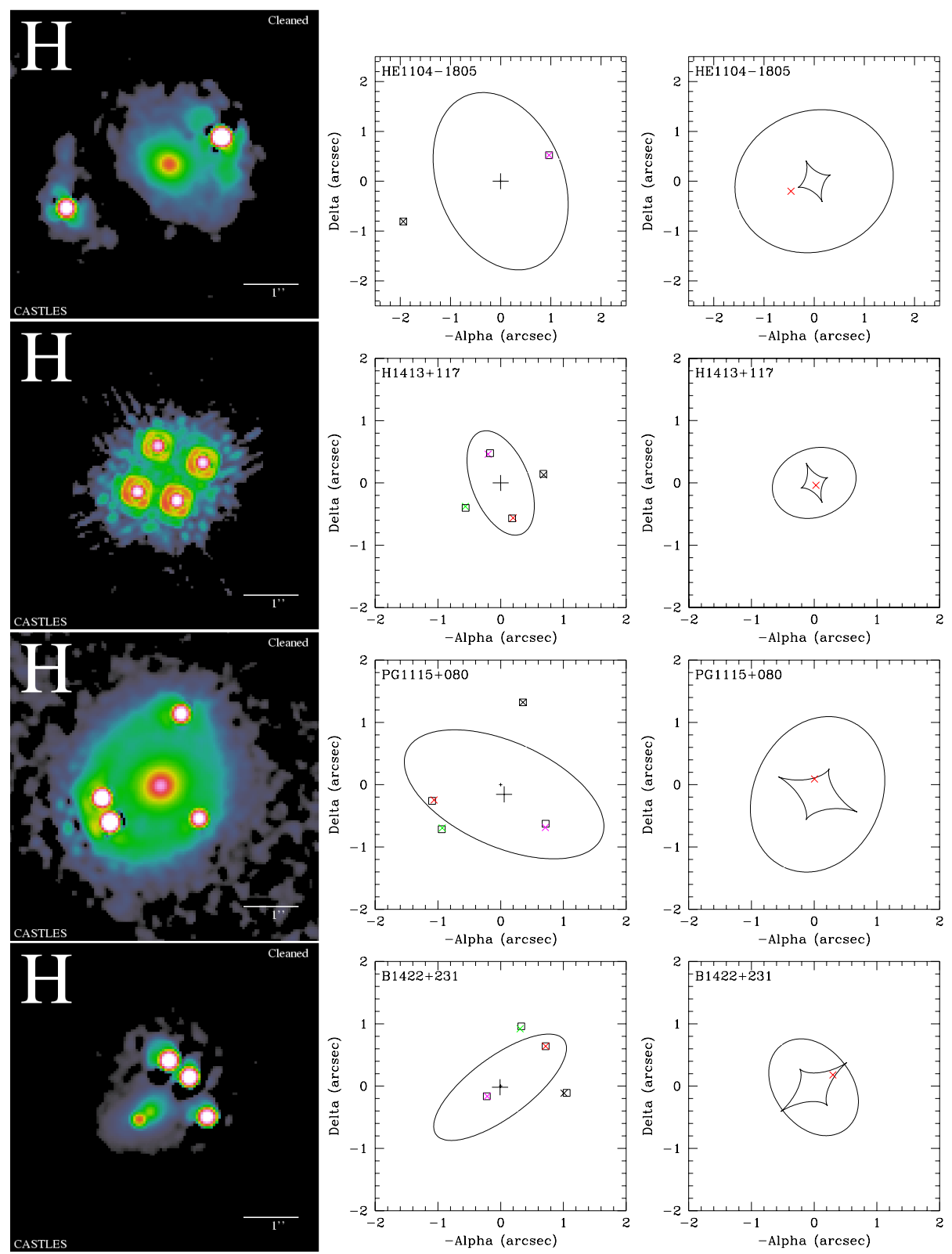

Fig. 10. Illustration of 4 representative multiply imaged quasars, as seen in the $\mathrm{H}$ band with HST (CfA-Arizona Space Telescope LEns Survey (CASTLES), left column). The central column is the best fit of an elliptical singular isothermal model (Kassiola and Kovner 1993, Korman et al. 1994; $\mathrm{x}=$ computed positions; squares and +: observed positions of lensed images and lensing galaxy). The right column shows the source position (x) and the caustics. Note the multiplicity and (a)symmetry of the images as a function of the source position with respect to the caustics; images are nearly merging when the source is close to the caustic. More information on these mirages may be found in Table 3 . 


\subsubsection{Caustics and amplification}

Both the source and the observer being very far from the deflector, gravitational lenses do not introduce any extra frequency shift in the spectral intensity of the lensed images in addition to the cosmological redshift. On the other hand, and in a first approximation, gravitational lenses are transparent. Therefore, the surface brightness of the lensed images is equal to that of the source (which, according to Liouville's theorem, is $I_{e} /(1+z)^{4}$ where $I_{e}$ is the surface brightness measured in the reference frame of the source and $z$ the cosmological redshift (e.g. Peebles 1993)). Conservation of the surface brightness can be appreciated by comparing the images of the coins located at the bottom of the swimming pool (Fig. 8).

As a consequence, the flux of a lensed image is amplified with respect to that of an infinitesimal source by a quantity $A$ equal to the ratio of the solid angle $\mathbf{d} \boldsymbol{\theta}$ of the magnified image to that $\mathbf{d} \boldsymbol{\theta}_{\mathbf{s}}$ of the real source:

$$
A(\boldsymbol{\theta})=\frac{\mathbf{d} \boldsymbol{\theta}}{\mathbf{d} \boldsymbol{\theta}_{\mathbf{s}}} \equiv \frac{1}{J(\boldsymbol{\theta})},
$$

where $J$ is given by the first equalities of relation (16). Thus, formally, an infinitesimal source located on a caustic is infinitely amplified. On the other hand, finite, transparent and non singular lenses always produce at least one magnified image (i.e. with $A>1$; Schneider 1984). For that reason, gravitational lenses also act as natural telescopes.

If the deflector has circular symmetry, relation (18) simply reduces to:

$$
A(\theta)=\frac{\theta d \theta}{\theta_{s} d \theta_{s}} .
$$

Since the gradient of $\alpha$ is always infinite at the center for singular models (see Eq. (8)), one easily sees that for such models the central image $(\theta=0)$ is always infinitely deamplified ${ }^{6}$. The lack of central images in observed gravitational lenses tends to show that the mass distribution of the lensing galaxies is strongly peaked at the center (Wallington and Narayan 1993).

As an application of relation (19), the flux amplification of a lensed image formed at position $\theta$ by a point mass is:

$$
A(\theta)=\frac{\theta^{4}}{\theta^{4}-\theta_{E}^{4}}
$$

The positions of the images being given by Eq. (11), the total amplification of the source can be found for a given value of $\theta_{s}$ :

$$
A\left(\theta_{s}\right)=\frac{\theta_{s}^{2}+2 \theta_{E}^{2}}{\theta_{s} \sqrt{\theta_{s}^{2}+4 \theta_{E}^{2}}} .
$$

\footnotetext{
${ }^{6}$ For circular lens models, a graphical approach of the lensing equation is useful to qualitatively derive the number, shape and amplification of the lensed images (see Refsdal \& Surdej 1994).
} 


\subsection{Time delays}

The light travel time $t$ from the source $S$ to the observer $O$ depends on the geometrical length of the trajectory and on the apparent light speed along the path, as measured by the observer. It can be derived from the expression of the static, weak field metric (Eq. (1) with $\left.\phi / c^{2} \ll 1\right)$ for photon trajectories $\left(d s^{2}=0\right)$ or from the analogy with optical refraction (Eq. (2)):

$$
c t=\int_{O}^{S} d l-\int_{O}^{S} \frac{2 \phi}{c^{2}} d l,
$$

where $d l=\sqrt{d x^{2}+d y^{2}+d z^{2}}$. The two terms on the right side of Eq. (22) are called the geometrical and the "potential" contributions, $t_{\text {geom }}$ and $t_{\text {pot }}$, respectively (Cooke and Kantowski 1975). Except when a symmetric lens is perfectly aligned with the source and the observer, the light travel time is different along each light trajectory (corresponding to each individual image) since the geometrical length and the encountered potential are different. Thus, if a signal is emitted by the source (i.e. a flash or some flux variations), time delays will separate its detection in each of the multiple images (Refsdal 1964).

To be more quantitative, it is easier to compute the time delay with respect to the pure geometrical case without any lens. Let us first assume a static euclidian space and consider both geometrical and potential contributions. From Fig. 4 and the lens equation (3) and again assuming $\alpha \ll 1$, one can derive:

$$
c \Delta t_{\text {geom }}=\frac{1}{2} \frac{D_{O D} D_{O S}}{D_{D S}}\left(\boldsymbol{\theta}-\boldsymbol{\theta}_{\mathbf{s}}\right)^{2} .
$$

The potential delay can be explicitly computed at first order by integrating the second term of equation (22) along the undeflected ray. For a point mass, $\phi$ is given by Eq. (7) and we find:

$$
c \Delta t_{p o t}=-\frac{4 G M}{c^{2}} \ln b+\mathrm{C},
$$

where $b$ is the impact parameter and $\mathrm{C}$ is a constant depending only on the distances between the observer, the lens and the source. Generalizing Eq. (24) into

$$
c \Delta t_{\text {pot }}=-P(\mathbf{b})+\mathrm{C},
$$

where $P$ represents a "deflection potential" and using Eqs. (22) and (6), we obtain:

$$
\boldsymbol{\alpha}(\mathbf{b})=\nabla_{\mathbf{b}} P(\mathbf{b}) .
$$

Comparing Eqs. (24) and (25) for the case of a point mass lens yields:

$$
P(b)=\frac{4 G M}{c^{2}} \ln b,
$$

and for a general surface mass density distribution:

$$
P(\mathbf{b})=\frac{4 G}{c^{2}} \int_{\mathcal{S}} \Sigma\left(\mathbf{b}^{\prime}\right) \ln \left|\mathbf{b}-\mathbf{b}^{\prime}\right| d b_{1}^{\prime} d b_{2}^{\prime} .
$$


Given the relations (23) and (25) and including the factor $\left(1+z_{D}\right)$ due to the expansion of the Universe ${ }^{7}$, the time delay can be rewritten as:

$c \Delta t=c\left(1+z_{D}\right)\left(\Delta t_{g e o m}+\Delta t_{p o t}\right)=\left(1+z_{D}\right)\left[\frac{1}{2} \frac{D_{O D} D_{O S}}{D_{D S}}\left(\boldsymbol{\theta}-\boldsymbol{\theta}_{\mathbf{s}}\right)^{2}-P(\mathbf{b})\right]+C$,

where $D_{O D}, D_{O S}$ and $D_{D S}$ represent angular cosmological distances.

In practice, time delays can only be measured between a pair of physically observed images. The delay between the flux variations in image 1 and image 2 is then simply $\Delta t_{12}=\Delta t_{1}-\Delta t_{2}$. The positions of the lensed images are related together through the lens equation (3) so that $\boldsymbol{\theta}_{\mathbf{s}}$ can be eliminated in Eq. (29). We find:

$$
\begin{aligned}
c \Delta t_{12}= & \left(1+z_{D}\right)\left[\frac{1}{2} D_{O D}\left(\boldsymbol{\theta}_{\mathbf{1}}-\boldsymbol{\theta}_{\mathbf{2}}\right)\left(\boldsymbol{\alpha}\left(D_{O D} \boldsymbol{\theta}_{\mathbf{1}}\right)+\boldsymbol{\alpha}\left(D_{O D} \boldsymbol{\theta}_{\mathbf{2}}\right)\right)-\right. \\
& \left.\left(P\left(D_{O D} \boldsymbol{\theta}_{\mathbf{1}}\right)-P\left(D_{O D} \boldsymbol{\theta}_{\mathbf{2}}\right)\right)\right] .
\end{aligned}
$$

It is interesting to note that the time delay does not depend explicitly on $H_{o}$. Indeed, from Eqs. (26) and (28) and with $\omega_{D}$ being the solid angle of the deflector, we have:

$$
\begin{aligned}
D_{O D} \boldsymbol{\alpha}\left(D_{O D} \boldsymbol{\theta}\right) & =\boldsymbol{\nabla}_{\boldsymbol{\theta}} P\left(D_{O D} \theta\right) \\
P\left(D_{O D} \theta\right) & =2 R_{s}\left[\int_{\omega_{D}} \sigma\left(\boldsymbol{\theta}^{\prime}\right) \ln \left|\boldsymbol{\theta}-\boldsymbol{\theta}^{\prime}\right| d \boldsymbol{\theta}^{\prime}+C\right]=2 R_{s} \tilde{P}(\boldsymbol{\theta}),
\end{aligned}
$$

where

$$
\sigma(\boldsymbol{\theta})=\Sigma\left(D_{O D} \boldsymbol{\theta}\right) \frac{D_{O D}^{2}}{M_{t o t}}
$$

and

$$
R_{s}=\frac{2 G M_{t o t}}{c^{2}}
$$

are the relative surface mass distribution $\sigma$ within $d \boldsymbol{\theta}$ and the Schwarzschild radius $R_{s}$, respectively. They are independent of $H_{o}$.

Inserting the latter results into Eq. (30), the time delay can be rewritten in a very simple form:

\footnotetext{
7 The origin of the factor $\left(1+z_{D}\right)$ in the potential delay is intuitive: to first order, the effects of $\phi$ (deflection and apparent slowing down of light) are confined near the deflector plane; because of the expansion, the time delay produced at $z_{D}$ is longer by a factor $\left(1+z_{D}\right)$ for an observer at $z=0$. Concerning the geometrical delay, one should note that it is much smaller than the expansion time $H_{o}^{-1}$ of the Universe, so $c \Delta t_{g e o m}=R_{o} \Delta \chi$, where $R_{o}$ is the present scaling factor and $\chi$ is the comoving distance in the FLRW models. $\Delta \chi$ between the deflected and undeflected rays can be computed with the relations in flat, spherical or hyperbolic triangles. The factor $\left(1+z_{D}\right)$ then arises after transforming the comoving distances into angular ones.
} 


$$
c \Delta t_{12}=2 R_{s}\left(1+z_{d}\right)\left[\frac{1}{2}\left(\boldsymbol{\theta}_{\mathbf{1}}-\boldsymbol{\theta}_{\mathbf{2}}\right)\left(\boldsymbol{\nabla}_{\boldsymbol{\theta}} \tilde{P}\left(\boldsymbol{\theta}_{\mathbf{1}}\right)+\boldsymbol{\nabla}_{\boldsymbol{\theta}} \tilde{P}\left(\boldsymbol{\theta}_{\mathbf{2}}\right)\right)-\left(\tilde{P}\left(\boldsymbol{\theta}_{\mathbf{1}}\right)-\tilde{P}\left(\boldsymbol{\theta}_{\mathbf{2}}\right)\right)\right] .
$$

Equation (33) shows that if we know the exact surface mass distribution, the positions of the multiple images with respect to the lens and the lens redshift, then the time delay measurement yields the total mass of the lens, irrespective of the values of the cosmological parameters $\left(H_{o}\right.$, etc...) or the source redshift. Once the deflector mass is known, the Hubble constant can be extracted, within the adopted lens model, from the observed angular separation of the lensed images (see Section 2.2.1).

As an illustration, in the case of the point mass lens model $\tilde{P}(\theta)=\ln \theta$ and

$$
c \Delta t_{12, P M}=2 R_{s}\left(1+z_{D}\right)\left[\frac{\theta_{2}^{2}-\theta_{1}^{2}}{2\left|\theta_{1} \theta_{2}\right|}+\ln \left|\frac{\theta_{2}}{\theta_{1}}\right|\right] .
$$

Depending on the exact configuration of the lensed images, the expected time delay ranges between a few days and a few months for a $10^{11} \mathrm{M}_{\odot}$ deflector and it scales as the deflector mass (i.e. as $R_{s}$ ).

\subsection{What is a good gravitational lens?}

An astrophysical object is a good lens if it is capable to form strongly distorted and/or multiple images of a distant source. Its surface mass density must locally be larger than the critical surface mass density $\Sigma_{\text {crit }}$ in order to produce an averaged surface mass density just equal to $\Sigma_{\text {crit }}$ between the lensed images (see Eq. (15)). On the other hand, since the angular separation between lensed images is about $2 \theta_{E}$ (see Eq. (14)), the latter will be resolved if the lens is sufficiently massive or close enough to the observer (see Eq. (13)) and if $\theta_{E}>\theta_{R}$ where $\theta_{R}$ is the object apparent size. Fig. 11 and Table 1 illustrate the quality of known compact astrophysical objects to act as gravitational lenses, given their total mass, distance and apparent size. Solar planets, globular clusters and local galaxies are too extended; exoplanets and extragalactic stars lead to typical angular separations of one micro-arcsecond (they are refered to as microlenses), that are well below the current instrumental resolution. Galactic stars, distant elliptical galaxies and rich galaxy clusters or central parts of clusters are good gravitational lenses.

\subsection{How to search at best for cosmic mirages?}

In order to discover cosmic mirages, two different strategies may be followed: selecting good lenses or selecting good sources.

\subsubsection{Selecting good lenses}

According to the last column of Table 1, the best gravitational lenses consist of dense galaxy clusters, since the angular region enclosed by the caustics (proportional to the square of the scaling factor $\theta_{E}$ ) is the largest. They are more 


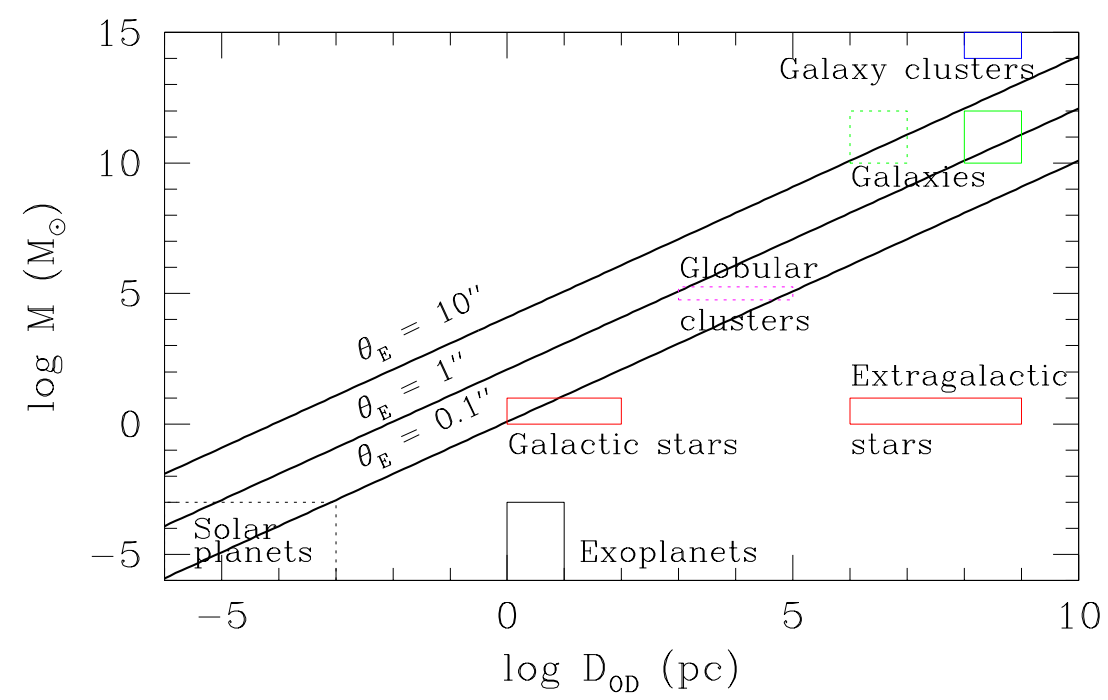

Fig. 11. Mass within the Einstein radius versus deflector angular distance for different values of $\theta_{E}$ (thick lines). Distance and total mass ranges are considered for various massive astrophysical objects; the sources are assumed to be at infinity (thin solid lines if $\theta_{R} / \theta_{E}<1$ and dotted lines otherwise, see Table 1).

efficient at redshifts in the range $0.3-0.5$ (see Fig. 6). Giant arcs are produced by the merging of strongly distorted images of an extended source intersecting or located inside the caustics. On the other hand, sources located outside the caustic are weakly distorted in arclets by the lens shear. Observations must be designed to detect distant objects with a very high space density (or integrated luminosity function), such as faint blue galaxies (Tyson 1988). Ultra-deep exposures of galaxy clusters through the B filter are clearly optimal for that purpose. Using the swimming pool analogy, this is equivalent to count as many coins as possible with the hope that some of them lie close to a caustic. Giant arcs and arclets are used as a diagnostic of the cluster mass distribution. For more details,

Table 1. Physical properties of potential strong gravitational lenses. $\theta_{R}$ is the apparent angular radius. The last column represents the angular cross section for lensing; brackets indicate that the resulting lensed images cannot be seen $\left(\theta_{R}>\theta_{E}\right)$.

\begin{tabular}{lccccc}
\hline Objects & Mass $\left(\mathrm{M}_{\odot}\right)$ & Radius $(\mathrm{pc})$ & $D_{O D}(\mathrm{pc})$ & $\theta_{R} / \theta_{E}$ & $\begin{array}{c}\pi \theta_{E}^{2} \\
\left(\operatorname{arcsec}^{2}\right)\end{array}$ \\
\hline \hline Solar planets & $10^{-6}-10^{-3}$ & $10^{-10}-10^{-9}$ & $10^{-6}-10^{-3}$ & $>40$ & {$[1]$} \\
Exoplanets & $10^{-6}-10^{-3}$ & $10^{-10}-10^{-9}$ & $1-10$ & 0.1 & $10^{-7}$ \\
Nearby stars & $1-10$ & $10^{-8}-10^{-6}$ & $1-100$ & $10^{-2}-10^{-3}$ & $10^{-2}$ \\
Extragalactic stars & $1-10$ & $10^{-8}-10^{-6}$ & $10^{6}-10^{9}$ & $10^{-6}-10^{-5}$ & $10^{-8}$ \\
Globular clusters & $10^{5}$ & $1-10$ & $10^{3}-10^{5}$ & $>300$ & {$[1]$} \\
Local galaxies & $10^{10}-10^{12}$ & $10^{3}-10^{4}$ & $10^{6}-10^{7}$ & $10-50$ & {$[20]$} \\
Distant galaxies & $10^{10}-10^{12}$ & $10^{3}-10^{4}$ & $10^{8}-10^{9}$ & $0.5-2$ & 20 \\
Galaxy clusters & $10^{14}-10^{15}$ & $10^{6}$ & $10^{8}-10^{9}$ & $1-100$ & $10^{3}$ \\
\hline
\end{tabular}


see the review papers on arcs and arclets by Fort and Mellier (1994) and Hattori et al. (1999).

On the other hand, selecting good lenses like galaxy clusters enhances the chance to resolve very distant background galaxies or AGNs located nearly on the same line-of-sight and which would otherwise be angularly too small to be studied at all. Indeed, as already mentioned in Section 1.5.2, a gravitational lens helps in significantly magnifying the regions of a background resolved source and thus, increases the effective angular resolution of the observations. This was first pointed out by Zwicky in 1937. The discovery of a young galaxy at $z=4.92$, located behind the cluster CL1358+62, has recently been reported by Franx et al. (1997). Several independent teams have also used cluster-lenses as gravitational telescopes, either with ISOCAM onboard the ISO satellite or using very sensitive detectors at the focus of $8-10 \mathrm{~m}$ telescopes, in order to detect optical, near- and mid-IR sources intrinsically fainter than those directly recordable outside the cluster (Altieri et al. 1999, Barvainis et al. 1999, Metcalfe et al. 1999, Pello et al. 1999). In addition, using both the magnification by foreground galaxy clusters and the high angular resolution of HST, it has been possible to resolve the stellar populations in a $z=4$ lensed galaxy (Bunker et al. 2000) and to study the star formation rate at very high redshifts (Bézecourt et al. 1999, Franx et al. 1997).

We shall not further explore here this approach of selecting lenses, as our review mainly deals with gravitational lensing among quasars.

\subsubsection{Selecting good sources}

If some luminous sources are a priori more likely to be lensed than others, they should be selected in order to discover new mirages. Let us assume for simplicity a non evolving population of lenses with mass $M$, whose comoving space density is thus a constant $n_{o}(M)$. The optical depth $\tau$ for lensing a source located at redshift $z_{s}$ is:

$$
\tau\left(z_{s}, M\right)=V_{\text {eff }}\left(z_{s}, M\right) n_{o}(M),
$$

where $V_{\text {eff }}$ is the effective proper volume in which the lens must be located in order to produce an observable gravitational mirage (Nemiroff 1988). "Observable" means that the angular separation and the magnitude difference between the multiple images can be resolved with the instrument used for the observations. $V_{\text {eff }}$ simply results from the integration of the effective cross section $\Sigma_{\text {eff }}$ along the line-of-sight:

$$
V_{\mathrm{eff}}\left(z_{s}, M\right)=\int_{0}^{z_{s}}(1+z)^{3} \Sigma_{\mathrm{eff}}\left(z, M ; z_{s} ; p_{i}\right) \frac{c d t}{d z} d z
$$

where the $p_{i}$ are the lens parameters.

The line-of-sight element $c d t / d z$ is given in the FLRW cosmologies by the following relation (e.g. Kayser et al. 1997):

$$
\frac{c d t}{d z}=\frac{c}{H_{o} \sqrt{1+z}} \frac{1}{\sqrt{(1+z)^{3} \Omega_{o}-(1+z)^{2}\left(\Omega_{o}+\lambda_{o}-1\right)+\lambda_{o}}} .
$$


If the instrumental angular selection function $S$ is equal to 1 for the only observable configurations of multiple images ${ }^{8}$ and zero otherwise, if $\mathbf{y}=\boldsymbol{\theta}_{\mathbf{s}} / \theta_{E}$ and if $p_{i}$ are the parameters of the lens model, the effective cross section is

$$
\Sigma_{\text {eff }}\left(z, M ; z_{s} ; p_{i}\right)=\left(D_{O D} \theta_{E}(z, M)\right)^{2} \iint_{\mathbb{R}^{2}} S\left(\mathbf{y}, z ; p_{i}\right) d y_{1} d y_{2} .
$$

Since the space density $n$ scales as $h^{3}$ or $h^{2}$ depending on whether it is derived from local counts or from a cosmological density in FLRW models, Eqs. (35), (36), (37), (38) and (13) easily reveal that $\tau \propto h^{\frac{2-2 \beta}{2-\beta}}$ or $\tau \propto h^{\frac{-\beta}{2-\beta}}$. Thus, the optical depth is independent of $H_{o}$ either for the SIS lens model $(\beta=1)$ in the first case or for the point mass model $(\beta=0)$ in the second hypothesis.

On the other hand, the total flux coming from multiple images is always amplified by a certain factor $A_{\text {tot }}$ with respect to the source flux (see Section 1.5). Therefore, the probability that a source with redshift $z_{s}$ and magnitude $b_{s}$ is a lensed object is not simply $\tau\left(z_{s}, M\right)$ but is given by:

$$
P\left(z_{s}, b_{s}\right)=\frac{N_{L}\left(z_{s}, b_{s}\right)}{N_{s}\left(z_{s}, b_{s}\right)}=\frac{\tau\left(z_{s}\right) N_{s}\left(z_{s}, b_{s}+2.5 \log A_{\mathrm{tot}}\right)}{N_{s}\left(z_{s}, b_{s}\right)}=\tau\left(z_{s}\right) \operatorname{Bias}\left(z_{s}, b_{s}\right)
$$

where the number-magnitude relation $N_{\mathrm{L}}\left(z_{s}, b_{s}\right)$ (resp. $N_{\mathrm{s}}\left(z_{s}, b_{s}\right)$ ) represents the number of multiply imaged sources (resp. unlensed sources) at redshift $z_{s}$ and blue magnitude $b_{s}$ per square degree and per magnitude interval; the last equality defines the so-called amplification bias ${ }^{9}$.

Usually, faint sources are more numerous than bright ones, so that the amplification bias is larger than 1. Moreover, if the logarithmic slope increases towards bright magnitudes, we expect the proportion of mirages to be larger at bright magnitudes.

Therefore, relations (35) and (39) show that the most likely lensed sources are

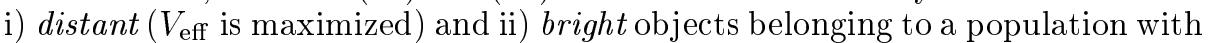
a steep number-magnitude relation at the observed wavelength (the amplification bias is then high; Turner 1980).

Highly Luminous Quasars (HLQs; $M_{V} \leq-27.5$ for $H_{o}=50 \mathrm{~km} / \mathrm{s} / \mathrm{Mpc}$ ) have been recognized long ago as excellent targets to search for new gravitational lenses because they are both distant and bright sources (Turner et al. 1984, Surdej et al. 1988b). Their number-magnitude relation has a steep bright end with a break around $b=19.2$ (Boyle et al. 1988, Hartwick \& Schade 1990). They have been selected to efficiently search for gravitational lenses by several teams; successful results are presented in Section 3. However, optical HLQ

\footnotetext{
8 More concretely, the angular selection function $S$ defines a "pseudo-caustic" into which the source must be located for its resulting multiple images to be detectable. For symmetric lens models, the detection criteria define the radius of the "pseudo-caustic" for each value of the lens redshift.

9 The amplification $A_{\text {tot }}$ depends on the source position $\mathbf{y}$, so that the amplification bias is generally merged into a new definition of the effective cross section and Eq. (38) becomes: $\Sigma_{\text {eff }}\left(z, M ; z_{s}, b_{s} ; p_{i}\right)=\frac{\left(D_{O D} \theta_{E}(z, M)\right)^{2}}{N_{s}\left(z_{s}, b_{s}\right)} \iint_{\mathbb{R}^{2}} N_{s}\left(z_{s}, b_{s}+2.5 \log A_{\text {tot }}\left(\mathbf{y} ; p_{i}\right)\right) S\left(\mathbf{y}, z ; p_{i}\right) d y_{1} d y_{2}$
} 
samples might suffer from dust extinction: extinguished secondary images or extremely reddened QSOs could remain undetected, resulting in incompleteness in the parent QSO sample and affected statistics. High resolution radio observations of flat spectrum radio sources escape this issue, but the lensed images are contaminated by double compact sources or by source structure (Helbig et al. 1999) and their redshift dependent number-magnitude relation is not very well known yet (Helbig et al. 1999, Falco et al. 1998). The amplification bias affecting flat spectrum radio sources seems to be lower than for HLQs. Finally, let us consider space X-ray observations. In that wavelength range, the simple source structure and the very rare spurious alignments with foreground galactic sources make the lens-candidate selection easy. On the other hand, the amplification bias is intermediate between optical and radio wavelengths. Unfortunately the expected detection rate remains small for Chandra simulations (Muñoz et al. 1999b).

\section{Astrophysical and cosmological applications}

It is now time to convince the reader that gravitational lensing among distant quasars does not just consist of an exotic or amazing consequence of General Relativity but that it does also provide excellent prospects for interesting cosmological and astrophysical applications. Two different kinds of approaches are possible. While statistics of multiple images in a complete sample of distant sources are needed to measure $\Omega_{o}$ and $\lambda_{o}$ and to bound the cosmological density of putative compact objects (Section 2.1), high quality observations of individual gravitational lens systems may lead to unique determinations of $H_{o}$ or of some astrophysical properties of the lens, the source and the intergalactic medium (Section 2.2).

\subsection{Statistical studies}

The observed number of multiply imaged QSOs in a sample of HLQs, but also, for each of them, the observed multiplicity of the lensed images, their angular separations, the possible detection of the lens and the measurement of its redshift provide useful information to constrain some cosmological- as well as some astrophysical parameters related to the lens model. Indeed, for example, the relative number of quadruply imaged QSOs increases with the ellipticity $\epsilon$ of the projected potential, the velocity dispersion $\sigma$ of the galaxies governs the mean angular separation between the lensed images and the number of observed mirages is a function of the number of galaxies, of the extinction and of the cosmological model (see below). On the theoretical side, statistics of gravitational lenses have first been established in detail by Turner, Ostriker and Gott in 1984. Further developments were performed by Fukugita et al. (1992) and Kochanek (1993a).

On the observational side, about 1200 HLQs have been imaged during the last ten years at optical wavelengths and more than 10 thousand sources have been investigated in radio surveys (see Section 3 ).

The first comparisons between the expected and the observed numbers of lenses were made by Maoz et al. (1993), Surdej et al. (1993a) and Kochanek 
(1993b) who was the first to apply the maximum likelihood analysis. They found that, as expected from theoretical models, approximately $1 \%$ of HLQs are multiply imaged by lensing galaxies (see also Section 3). However, there are degeneracies between some parameters and, although gravitational lensing may help in setting individual constraints on their values, it can also be combined with prior knowledge of some of the parameters (Kochanek 1996, Quast and Helbig 1999).

While the main results are discussed in Section 5, we explain here the interest of statistical lensing with two concrete examples.

\subsubsection{The cosmological parameters $\Omega_{o}$ and $\lambda_{o}$}

The fate of the Universe is intimately related to the values of the cosmological density parameter $\Omega_{o}$ and of the cosmological constant $\lambda_{o}$. Their determination constitutes one of the most important challenges in present observational cosmology. How can gravitational lensing help? $\Omega_{o}$ and $\lambda_{o}$ intervene in the computations of the angular distances and of the effective volume in which a lens produces multiple images of a source (see Eq. (39)). Thus, the expected number of multiply imaged QSOs in a large sample strongly depends on those parameters, as was first pointed out by Turner (1990) and Fukugita et al. (1990). Cosmological distances to a given redshift increase for lower values of $\Omega_{o}$ or for larger values of $\lambda_{o}$; this results in a degeneracy between these two parameters in most cosmological tests and gravitational lensing is not an exception. Fortunately, the influence of $\lambda_{o}$ on $V_{\text {eff }}$ is much stronger than that of $\Omega_{o}$ : whatever a reasonable value of $\Omega_{o}$, values of $\lambda_{o}$ close to 1 lead to the prediction of detecting many more lenses than is actually observed in optical samples. Gravitational lensing statistical studies thus yield a natural upper limit on the value of the cosmological constant. Fukugita et al. (1990) also showed that the mean redshift of the deflectors is larger if $\lambda_{o}$ dominates over $\Omega_{o}$.

\subsubsection{Space density of dark compact objects}

We saw in Section 1.7 that an infinitesimal source is necessarily multiply imaged by a foreground object located close to its line-of-sight, provided the latter one is sufficiently compact. The deflector does not need to be visible. Press and Gunn had already noted in 1973 that counting gravitational lensing events in a sample of distant sources would help in constraining the contribution $\Omega_{L}$ to the critical density of the Universe due to a putative cosmological population of dark compact objects. One can show from Section 1.3 that the angular separation $\Delta \theta$ between lensed images is about $10^{-6} \sqrt{M_{L} / \mathrm{M}_{\odot}}$ arcsec where $M_{L}$ is the mass of the dark compact extragalactic lens. Thus, a given instrumentation is capable to detect the lensing effects of compact objects in a relatively narrow range of masses (see Fig. 12). For example, ground based optical telescopes can reveal the effects of lenses with $M_{L}$ between $10^{10.5}$ and $10^{15} \mathrm{M}_{\odot}$ while a higher resolution and a smaller field make the radio observations more sensitive around $10^{6} \mathrm{M}_{\odot}$. Below $10^{5} \mathrm{M}_{\odot}$, the resulting multiple images cannot be resolved: this is the socalled milli- or microlensing range. However, the flux variations induced in the 

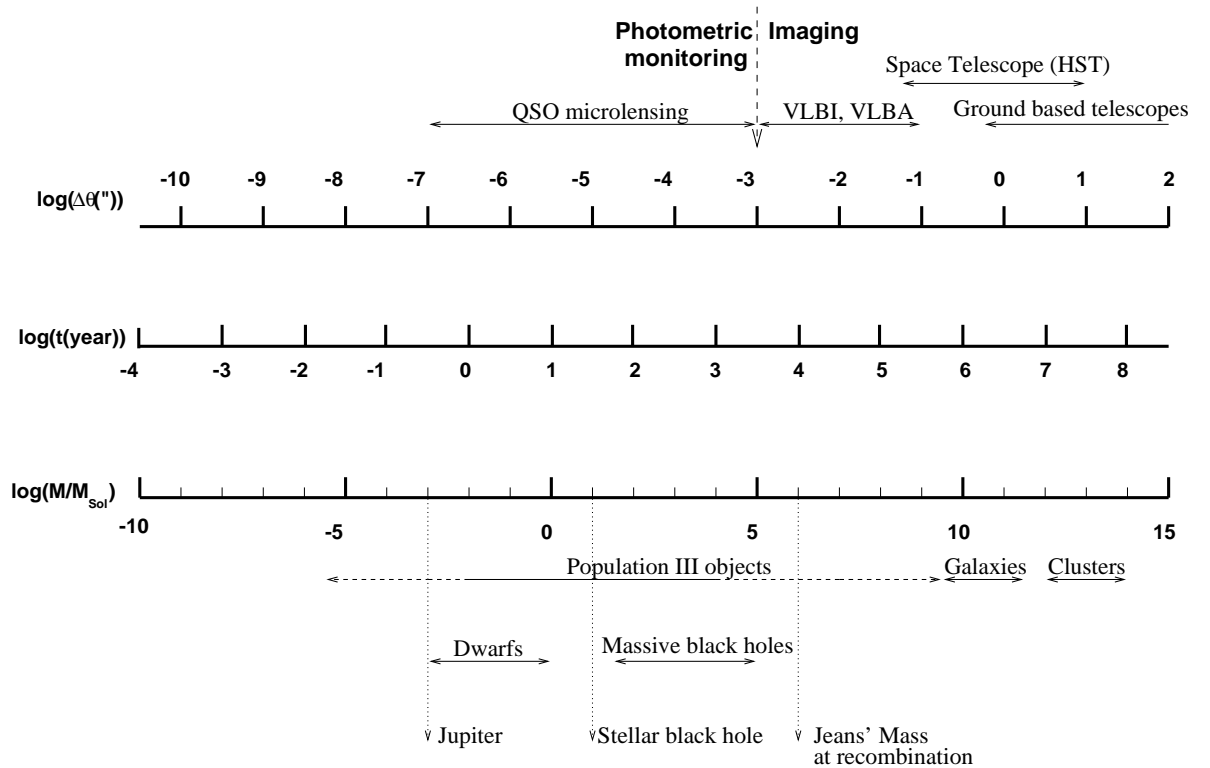

Fig. 12. Correspondence between the mass of a Schwarzschild lens located at a cosmological distance, the lifetime of the multiple images (assuming relative transverse velocities of 1000 $\mathrm{km} / \mathrm{s}$ ), their typical angular separation, the range of application of existing observational techniques and the possible astrophysical nature of the lens.

lensed images by the relative motion of a deflector with respect to the observersource direction can be recorded by means of a photometric monitoring (the amplification of the source increases when it gets closer to a caustic, see Eq. (21)). Note however that for a $10^{5} \mathrm{M}_{\odot}$ lens, the typical time scale of the event gets rather large ( $\sim 3,000$ years, see Fig. 12). Observed constraints are presented in Section 5.

Photometric variations due to microlensing are also observed locally, on stars located in the Magellanic Clouds or in the bulge of the Milk Way. These observations are used to constrain and to characterize the population of (dark) compact objects present in the halo and to investigate the structure of our Galaxy. For more details on this technique, see the review by Paczyński (1996) and references therein.

\subsection{Individual studies}

Detailed studies of individual multiply imaged QSOs do also lead to interesting probes for cosmology and astrophysics. We introduce below some of the most representative examples.

\subsubsection{Time delays and the measurement of $H_{o}$}

Refsdal (1964) was the first to point out that, in principle, the Hubble constant $H_{o}$ could be determined from gravitational lensing studies of distant sources, 
without any assumption on the existence of a standard candle. As the angular separation of multiple images $\Delta \theta \simeq 2 \theta_{E}$ is proportional to $\sqrt{M_{o} H_{o}}$ (see Eqs. (4), (13) and (14)), it is not possible to disentangle the effect of a larger deflecting mass combined with that of a lower value of $H_{o}$. The measurement of a time delay $\Delta t_{i, j}$ between flux variations of two lensed images $i$ and $j$ breaks the degeneracy, as the total mass can be derived from Eq. (33) provided that the mass distribution is known. More generally, one can see from relations (3), (9), (31) and (33) that $H_{o}$ is inversely proportional to the time delay as follows:

$$
\Delta t_{i, j}=H_{o}^{-1}\left(1+z_{D}\right) D\left(z_{D}, z_{s}, \Omega_{o}, \lambda_{o}\right) f\left(\boldsymbol{\theta}_{\mathbf{i}}, \boldsymbol{\theta}_{\mathbf{j}} \text {, lens model }\right),
$$

where $D=D_{O D} D_{O S} / D_{D S}$ and $f$ is a lens model dependent function of the observed image positions $\boldsymbol{\theta}_{\mathbf{i}}$ and $\boldsymbol{\theta}_{\mathbf{j}}$.

Therefore, the successful determination of $H_{o}$ relies on:

1. intensive photometric monitoring of a variable multi-imaged QSO in order to precisely determine $\Delta t_{i, j}$;

2. accurate observations of the positions $\boldsymbol{\theta}_{\mathbf{i}}$ of the lensed images with respect to the deflector;

3. good knowledge of the lens redshift $z_{D}$ and of the cosmological parameters. The error due to unknown precise values of $\Omega_{o}$ and $\lambda_{o}$ is not important as long as the lens redshift is moderate $\left(z_{D} \sim 0.5\right)$;

4. a very good estimate of the deflector mass distribution.

The last item represents the most difficult issue. A generic problem comes from the fact that the observed lensed image positions and flux ratios ${ }^{10}$ are functions of the deflection angle and of its first derivatives, respectively (see Eqs. (3) and (18)), while the time delay is a function of the deflection potential $P$, which is the integral of the deflection angle (see Eq. (26)). Thus, several different models can reproduce the observed lensed image positions and flux ratios but not the time delay, leading to different estimates of $H_{o}$. This is known as the degeneracy problem ${ }^{11}$. It has first been pointed out by Falco et al. (1985) and Gorenstein et al. (1988b). They noted that adding a dark sheet of matter with constant normalized surface mass density $\kappa=\Sigma / \Sigma_{\text {crit }}$ and reducing the lensing mass by a factor $(1-\kappa)$ does not affect the image configuration (positions and flux ratios) but reduces the predicted value of $H_{o}$ by a factor $1-\kappa$. Assuming $\kappa=0$ would yield an upper limit on the value of $H_{o}$.

Thus, additionnal observational constraints must be used whenever available to better determine the potential of the lens. The detection of lensed extended structures provide extra values for the derivatives of the potential and help in improving the model. Such extended structures may consist of radio jets, alike those detected with VLBI in the case of Q0957+561 (Porcas et al. 1981, Gorenstein et al. 1988a), or the quasar host galaxy observed with HST, e.g. Falco et al. (1997a) and Bernstein et al. (1997) for Q0957+561, but see additional cases in Section 5.3. On the other hand, mapping the velocity distribution in the deflector breaks the mass-sheet degeneracy. In the case of Q0957+561, the main lens is embedded in a galaxy cluster. The detection of a weak shear on faint background galaxies and/or the measurement of the velocity dispersion of

\footnotetext{
10 The flux ratio must be free of contamination by microlensing and/or dust absorption.

11 An extreme example is given by assuming that the deflector is a point mass in the case of Q0957+561 A\&B: the derived value of $H_{o}$ would then be $207 \mathrm{~km} / \mathrm{s} / \mathrm{Mpc}$ !
} 


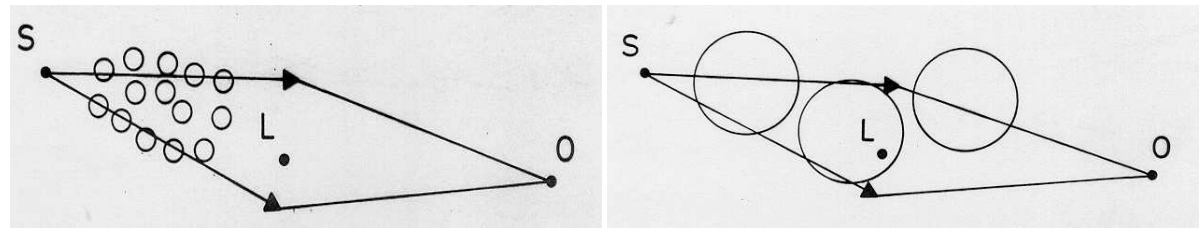

Fig. 13. Probing the size of absorbing intergalactic clouds with gravitational lensing. Small clouds usually affect only one line-of-sight (left) while large clouds often extend over the two lines of sight and create coincident absorption features (right).

the cluster of galaxies enable one to independently determine the contribution of $\kappa$ to the lensing potential $P$ and also to break the degeneracy.

Ultimately, only independent lens modelings based upon the most exhaustive set of observational constraints will attest the reliability of Refsdal (1964)'s proposal to measure $H_{o}$ and the size of the Universe. This important but difficult endeavor has driven much effort during the past years to improve our knowledge of known mirages and to discover new ones in larger samples.

\subsubsection{Size of intergalactic clouds}

Astrophysical objects can be detected not only by their emitted radiation but also by their absorption signature in the spectra of luminous background sources. Cold intergalactic clouds are revealed by their Ly $\alpha$ and/or metallic absorption lines (C IV, Mg II,...) seen in the spectra of distant quasars. If such a QSO is multiply imaged by gravitational lensing, the different lines of sight probe the clouds at various impact parameters. A determination of the numbers of coincident or anti-coincident absorption lines in high resolution spectra of the lensed components allows to constrain the size of the clouds (see Fig. 13): this is particularly interesting as some of these clouds might be connected with galaxy formation (e.g. Wolfe 1988).

\subsubsection{Extinction in lensing galaxies}

Lensing galaxies have long been thought to be transparent. This simplifying hypothesis was justified by the fact that deflected light rays avoid the most central parts of the deflector and have a typical impact parameter of 5-10 kpc. Moreover the most efficient lensing galaxies are the elliptical ones. However, extinction by dust located in the lens has been suspected from the differential reddening observed in some multiply imaged quasars, like Q2237+0305 (Yee 1988, Nadeau et al. 1991), MG0414+0534 (Hewitt et al. 1992, Lawrence et al. 1995, McLeod et al. 1998), MG1131+0456 (Larkin et al. 1994) or RXJ0911.4+0551 (Burud et al. 1998). On the other hand, some lenses are edge-on spiral galaxies (e.g. B1600+434, Jaunsen \& Hjorth 1997). The latter can produce strong lensing effects just by projection of the disk (Möller \& Blain 1998) but they are more dusty and the resulting mirages could mostly remain undetected (Bartelmann \& Loeb 1998).

Quantifying the effects of dust constitutes an important issue in statistical studies of gravitational lensing when dealing with optical samples of QSOs. The 
determination of $\lambda_{o}$ for example will be strongly affected if many multiply imaged QSOs are hidden by dust (see Section 5); this favours the use of radio samples for statistical purposes because dust has no effect at radio wavelengths, but we are then faced with the poor knowledge of the redshift-dependent luminosity function of radio sources (Falco et al. 1998, Helbig et al. 1999).

On the positive side, the observed differential reddening in multiply imaged QSOs may be used to determine the dust extinction law in very remote galaxies and compare it to what we know about the dust properties in the Milky Way, the Magellanic Clouds and some very nearby galaxies. This was first pointed out by Nadeau et al. (1991) for the case of Q2237+0305 and was further developed by Jean \& Surdej (1998) in order to retrieve the redshift of the lens, even if the latter is not directly detected. To achieve this, accurate spectrophotometry or photometric measurements through many passbands, ranging from UV to near IR, are needed for each lensed component.

\subsubsection{Microlensing and structure of the QSOs}

We saw in Section 1.7 that a given star located at a cosmological distance is not a very good lens because it has nearly no chance to be coaligned with a background source. However, when a QSO is lensed by a galaxy into multiple images, the probability that one of the stars belonging to the lens transits just in front of one of the macrolensed QSO images is much higher. The angular separation between the sub-images cannot be resolved $\left(10^{-6 \prime \prime}\right)$ but the variations of the integrated flux of the affected macro image can be recorded. The stellar microlens only quantitatively modifies the flux for very small sources, typically those with a size smaller than that of the caustic in the source plane: it acts as a magnifying glass whose space motion allows to scan very compact sources. Quasars are not angularly resolved with conventional telescopes. But, since microlensing is achromatic, it can be used to analyse the chromatic structure of quasars. Indeed microlensing preferentially amplifies the continuum emission region, coming from the central part of the QSO ( $\simeq 10^{-3} \mathrm{pc}$ corresponding to angular separations $<10^{-6 \prime \prime}$ ) while the broad emission line flux (emitting region $>1 \mathrm{pc}$ ) remains unchanged. Microlensing induces uncorrelated variations of macrolensed images of a QSO during a photometric monitoring. The best cases occur with nearby lenses, such as Q2237+0305, because of their larger angular size, of the larger value of the stellar Einstein radius, of the faster apparent motion of stars and because the time delay is only about 1 day (Lewis et al. 1998). Several other astrophysical uses of microlensed sources have been suggested: for instance, Agol and Krolik (1999) have investigated the possibility to image a quasar accretion disk using induced microlensing variability of a lensed QSO image.

\section{Surveys for multiply imaged quasars}

As stated in Section 1.8, the dependence of the probability $P\left(z_{s}, b_{s}\right)$ for a distant source to be lensed as a function of both its redshift $z_{s}$ (Eq. (35)) and its apparent magnitude $b_{s}$ (via the amplification bias in Eq. (39)) has prompted several groups to search for gravitational lens candidates in large samples of bright and distant quasars whose luminosity function is known to be very steep (Turner et al. 
1984, Surdej et al. 1988b). Highly Luminous Quasars (typically $M_{V} \leq-27.5$ for $H_{o}=50 \mathrm{~km} / \mathrm{s} / \mathrm{Mpc}$; hereafter HLQs) were the first such bright sources to be systematically imaged at high angular resolution with the aim of performing statistical studies.

In this section, we review most of the surveys for multiply imaged quasars that have been carried out using both optical and radio telescopes.

\subsection{Optical surveys}

Most of the HLQs selected in the different optical samples described hereafter were drawn from the catalogue of Véron-Cetty \& Véron $(1987,1991,1993)$ available at the time of the observations. As stated by Véron-Cetty \& Véron, their catalogue does not represent by any means a complete sample of quasars; it merely consists of a non exhaustive compilation of quasars that have been published up to a certain date in the scientific literature and which are affected by multiple and complex observational biases. One of these biases relates to the "publication effect" which is known to privilege the insertion of interesting or peculiar objects in a quasar catalogue. On the contrary, Kochanek (1991) has estimated that some $30 \%$ of multiply imaged quasars could have been missed in quasar samples because of the initial search criteria used to select QSOcandidates as point-like and blue objects. For instance, multiply imaged quasars reddened by the lens or displaying a complex image morphology have most likely been rejected as quasar candidates in the original samples. Futhermore, the apparent magnitudes listed in quasar catalogues also raise serious problems. Indeed, in order to properly estimate the amplification bias (cf. Eq. (39)), one should make use of an accurate estimate of the quasar apparent magnitude at the time of discovery. Unfortunately, most of the published quasar magnitudes only consist of rough estimates, very often based upon photographic plates that were used some time ago to search for the quasars. More recent and accurate photometric measurements are usually available but due to the photometric variability of quasars, it is quite unsafe to use the latter magnitudes in order to calculate the amplification bias.

Nevertheless, the Véron-Cetty \& Véron compilation of quasars has still the merit of providing a reliable and trustworthy set of observational data on quasars in general. It is now foreseen that on-going and future automated searches for quasars such as the Hamburg-ESO survey (Wisotzki et al. 1996b, Reimers and Wisotzki 1997), the Sloan Digital Sky Survey (Loveday 1996), the 2dF QSO redshift survey (Smith et al. 1999), the International 4-m Liquid Mirror Telescope (ILMT) project (Surdej and Claeskens 1997) or the proposed GAIA space experiment ${ }^{12}$ will produce large sets of complete, well controled and calibrated data which are absolutely necessary in order to accomplish further progress based upon gravitational lensing statistics.

We list below all individual quasar samples that we have used and merged hereafter to form a "final" optical catalogue of some 1164 distinct bright quasars for which gravitational lensing statistics has been applied in Section 5. All details about the quasars included in these individual samples may also be found in a

\footnotetext{
12 Information on GAIA space mission may be found at ESA's URL: http://astro.estec.esa.nl/GAIA
} 
long table published by Claeskens (1999); the table is also available through Internet $^{13}$.

\subsubsection{The ESO sample}

This sample of bright and distant quasars has been observed at ESO (La Silla) between 1989 and 1993 with the $3.6 \mathrm{~m}, 2.2 \mathrm{~m}$ and $1.5 \mathrm{~m}$ telescopes (Surdej et al. 1989). A total of 393 HLQs, essentially selected from the Véron-Cetty \& Véron catalogue of quasars have been imaged at high angular resolution and under good seeing conditions. The average observational characteristics of this and all subsequent quasar samples are summarized in Table 2. During the course of these observations, three new gravitational lens systems have been identified: Q1208+1011 A\&B (Magain et al. 1992), Q1009+025 A\&B (Surdej et al. 1993b) and J03.13 A\&B (Claeskens et al. 1996a, Surdej et al. 1997), and four known gravitational lens systems have been re-discovered: H1413+117 A-D (Magain et al. 1988), PG 1115+080 A-C (Weymann et al. 1980), UM673 A\&B (Surdej et al. 1987, 1988a) and HE1104-1805 A\&B (Wisotzki et al. 1993).

\subsubsection{The Crampton et al. CFHT sample}

A sample of 101 HLQs, also drawn from the Véron-Cetty \& Véron (1987) catalogue, has been observed with an active CCD camera at the prime focus of the $\mathrm{CFH}$ telescope in order to detect close separation gravitational lens systems (Crampton et al. 1992). In spite of the excellent image seeing conditions prevailing during the observations (average FWHM $\sim 0.7$ "), no new gravitational lens was identified.

\subsubsection{The Yee et al. CFHT sample}

Another set of 104 HLQs, selected from the Hewitt \& Burbidge (1987) catalogue of quasars, has also been imaged with the CFHT at high angular resolution by Yee et al. (1993). Among several identified lens candidates, H1413+117 was rediscovered but all other mutiple image systems turned out to be stars.

\subsubsection{The NOT sample}

The initial number of quasars imaged with the NOT at La Palma amounts to 168 (Jaunsen et al. 1995). These quasars, mainly located in the northern hemisphere, have been selected on the basis of their bright absolute magnitude listed in the Véron-Cetty \& Véron (1991) catalogue but also from an unpublished list of quasars by C. Hazard and from a preliminary list of bright quasars discovered by the Hamburg team (Jaunsen, private communication). The number of distinct quasars that have been observed with the NOT is now approximately 380. Several of these have shown an interesting and/or complex image morphology but no new lens has been identified among these.

\footnotetext{
13 The URL address is:

http://vela.astro.ulg.ac.be/themes/extragal/gravlens/bibdat/engl/table0.html
} 


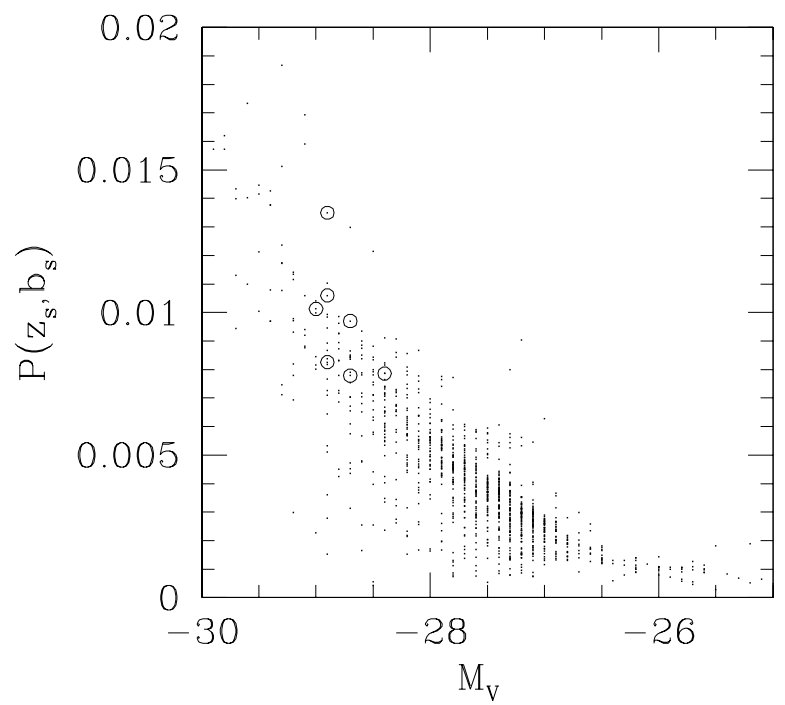

Fig. 14. Probability $P\left(z_{s}, b_{s}\right)$ for each QSO in the merged optical sample (see Tab. 2 and Section 3.1.6) to be multiply imaged as a function of its $V$ absolute magnitude. The seven known lensed quasars in that sample are encircled.

\subsubsection{The HST sample}

This sample consists of 494 HLQs from the Véron-Cetty \& Véron (1991) catalogue. They were observed in the snapshot mode with the Hubble Space Telescope (Maoz et al. 1993). Due to the observing policy with HST, known lenses were excluded from the sample. Only one new case of multiply imaged quasar has been found among the 494 HLQs: Q1208+1011 has been independently rediscovered with HST (Bahcall et al. 1992).

Table 2. Average observational characteristics of the different optical samples.

\begin{tabular}{|c|c|c|c|c|c|c|}
\hline QSO sample & $z_{s}$ & $b_{s}$ & $M_{V}$ & $\begin{array}{l}\text { FWHM } \\
\text { [arcsec.] }\end{array}$ & $N_{q}$ & $N_{q, \text { best }}(\%)$ \\
\hline $\begin{array}{l}\text { ESO } \\
\text { (Claeskens 1999) }\end{array}$ & 2.28 & 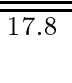 & $\overline{-27.8}$ & 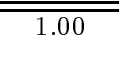 & $\begin{array}{l}393 \\
\end{array}$ & $\overline{\overline{266(68 \%)}}$ \\
\hline $\begin{array}{l}\text { CFHT } \\
\text { (Crampton et al. 1992) }\end{array}$ & 2.38 & 18.6 & -27.5 & 0.67 & 101 & $78(77 \%)$ \\
\hline $\begin{array}{l}\text { CFHT } \\
\text { (Yee et al. 1993) }\end{array}$ & 2.18 & 18.0 & -27.8 & 0.76 & 104 & $38(37 \%)$ \\
\hline $\begin{array}{l}\text { NOT } \\
\text { (Jaunsen et al. 1995) }\end{array}$ & 2.02 & 17.6 & -27.6 & 0.90 & 463 & $354(76 \%)$ \\
\hline $\begin{array}{l}\text { HST } \\
\text { (Maoz et al. 1993) }\end{array}$ & 2.17 & 18.1 & -27.5 & - & 494 & $428(87 \%)$ \\
\hline MERGED & 2.17 & 17.9 & -27.6 & 0.90 & 1555 & 1164 \\
\hline
\end{tabular}




\subsubsection{The merged sample}

As expected, quite a few quasars have been independently observed by the different teams. If we merge all previous optical samples, the resulting one does actually have 1555 observations for a total of 1164 distinct quasars. In case of duplication, we kept the most outstanding observation, i.e. the observation for which the probability of detecting a gravitational lens is the highest, usually corresponding to the best seeing conditions or characterized by the best angular selection function. Indeed, the non detection of multiple images for most of the HLQs also helps in constraining the lensing models and the strength of these constraints directly relates to the image quality of the observations. Table 2 summarizes the average observing conditions pertaining to the individual optical samples of quasars. It gives the mean redshift $z_{s}$, the mean blue magnitude $b_{s}$ and the resulting average absolute magnitude $M_{V}\left(H_{o}=50 \mathrm{~km} / \mathrm{s} / \mathrm{Mpc}, \Omega_{o}=1\right.$, $\lambda_{o}=0$ ) of the $N_{q}$ observed quasars (under an average seeing FWHM) in each individual sample. The $N_{q, \text { best }}$ column lists the final number (or percentage) of HLQs retained after elimination of the duplicated observations. Thanks to the higher angular resolution of the Hubble Space Telescope, this sample turns out to include the higher proportion $(87 \%)$ of retained observations, followed in numbers by the NOT and the ESO samples. Table 3 in Section 4 summarizes the observed properties of the seven multiply imaged quasars identified in the final optical sample of 1164 HLQs. Fig. 14 illustrates the a priori probability of detecting a gravitational lens for each of these quasars (see Eq. (39)), as a function of their $\mathrm{V}$ absolute magnitude. In accordance with theoretical expectations (see Section 1.8.2), the seven known lenses are indeed identified among the most luminous HLQs.

\subsubsection{Additional optical samples}

For the sake of completeness, we should like to mention the existence of several additional optical surveys for multiply imaged quasars among HLQs.

Since 1990, the Hamburg/ESO survey (HE quasars) has sampled the bright quasar population $(B<17.5$ and $z<3.2)$ over a substantial area of the southern sky (Wisotzki et al. 1996b, Reimers et al. 1996, Engels et al. 1998). Similarly, gravitational lenses have recently been detected among bright quasars identified in the Second Byurakan Spectral survey (SBS quasars) for bright $(B<19.5)$ emission-line objects (Chavushyan et al. 1997). Direct imaging of bright quasars has also been reported in the FKS survey (Kochanek et al. 1995). Some interesting gravitational lens systems discovered in these bright QSO samples have been definitely confirmed and are also listed in Table 3 .

Ratnatunga et al. (1995, 1999) have searched for multiply imaged sources (double images, quads, arcs, ...) around foreground galaxies, at sub-arcsec angular scales, on parallel recorded WFPC2 CCD frames obtained in the context of the HST Medium Deep Survey (MDS). Although the nature of most of the very faint sources remains to be determined on the basis of deep spectroscopic observations, they have set up a list of the top 10 good gravitational lens candidates (see Table 3 ). The first of these (HST14176+5226) has been confirmed to consist of 4 multiple images of a faint quasar at $\mathrm{z}=3.4$ lensed by a foreground galaxy 
at $\mathrm{z}=0.809$ (Crampton et al. 1996). It is probable that more faint quasars will be identified among the remaining MDS gravitational lens candidates.

Finally, Scarpa et al. (1999) have reported evidence for macro-lensing effects for at least 4 targets in their HST WFPC2 Snapshot Survey of BL Lac objects: $1 \mathrm{ES} 0033+595,1 \mathrm{ES} 0502+675,1 \mathrm{ES} 1440+122$ and H1517+656. If some of these candidates prove to be real lenses, they may constitute good probes to determine $H_{o}$. Indeed, BL Lac objects are known to be highly photometric variable. Furthermore, they are likely to be superluminal sources, offering a unique possibility to map the image/source magnification (cf. Eq. (21)) radially across the source plane in just a few years, providing strong additional constraints on the lens model. See also Lewis and Ibata (1999) for the case of PKS0537-441 (no evidence for lensing).

\subsection{Radio surveys}

In addition to the very first MG-VLA radio survey that has led to the discovery of several famous lenses (Lawrence et al. 1986, see also Table 3 for a complete list), the largest systematic radio search is the on-going CLASS (Cosmic Lens All Sky Survey, Myers et al. 1995). This very ambitious survey is the successor of the pioneering JVAS (Jodrell Bank - VLA Astrometric Survey, Patnaik et al. 1992, King et al. 1999) which was aimed at the identification in the northern sky $\left(0^{\circ}<\delta<75^{\circ}\right)$ of new lenses among flat-spectrum radio sources (i.e. with $\alpha>-0.5$ for the radio flux $f_{\nu} \sim \nu^{\alpha}$ between $5 \mathrm{GHz}$ and $325 \mathrm{MHz}$ ). Radio sources with a flux density greater than $200 \mathrm{mJy}$ at $5 \mathrm{GHz}$ were selected for JVAS, against $25 \mathrm{mJy}$ for CLASS. Criteria to select the gravitational lens candidates are multiple images with angular separations in the range $0.3^{\prime \prime}-6^{\prime \prime}$ and flux ratios $<20$ (King et al. 1999).

JVAS and CLASS consist of well defined surveys for which VLA radio images at $8.4 \mathrm{GHz}$ with $0.2^{\prime \prime}$ resolution have already been obtained for approximately 15,000 flat-spectrum radio sources (i.e. approximately 3,000 for JVAS $+12,000$ for CLASS; Myers et al. 1999).

Flat-spectrum radio sources are particularly well suited as a population from which one can obtain, in principle, unbiased samples of interesting gravitational lenses (Helbig et al. 1999). Indeed, the majority of flat-spectrum radio sources are likely to be single compact objects. It is thereby easy to identifiy lens candidates from those VLA maps which display multiple nearby flat-spectrum radio components. In addition, flat-spectrum radio sources often turn out to be variable and thus constitute prime candidates for a determination of $H_{o}$ from the measurement of time delays.

From an inspection of the JVAS-CLASS VLA images, some 150-300 gravitational lens candidates seem to have been identified (Fassnacht et al. 1999b). These were then imaged at higher angular resolution with the MERLIN interferometric array and sometimes with the VLBA. There remained of the order of 40 likely candidates for which additional observations were taken at optical and IR wavelengths using either the William Herschel Telescope on La Palma, the Hubble Space Telescope, or the Keck Telescope on Mauna Kea. More than a dozen of these candidates have already proven to be real gravitational lenses (Myers et al. 2000), the HST direct images often revealing the faint deflecting galaxy. 
Among the most famous gravitational lens systems detected in the JVAS/ CLASS survey (see Table 3 for an exhaustive list), we may note B0218+357 (a good candidate to estimate $H_{o}$ from the measurement of the time delay) and B1600+434 (the reddening of the faint quasar image is outstanding), both with as lens a spiral galaxy, almost seen edge-on in the latter case. The CLASS lens system B1608+656 corresponds to a quadruply imaged post-starburst radio galaxy. HST observations have enabled to resolve both the galaxy source and the lens. This system has been monitored with the VLA. Three independent time delays have been derived and an estimate of $H_{o}$ has been proposed (Fassnacht et al. 1999b, see Section 5.3). B1933+503 shows, at sub-arcsecond angular scales, a total of up to 10 lensed radio images originating from different parts of an active radio galaxy (both the compact flat-spectrum radio core and one of the steep spectrum radio lobes are quadruply imaged whereas the second steep spectrum radio lobe is only doubly imaged, King et al. 1997).

The real advantages of gravitational lens radio surveys over optical ones is that the radio-emitting regions of the quasar sources ought to be substantially larger than the optical ones, such that microlensing by stars in the deflector should not affect the observed flux ratios. These radio flux ratios may then be used to better constrain the lens model. Furthermore, extinction effects are totally negligible.

In spite of this, the observed flux ratios observed in the radio for several JVAS/CLASS systems such as the very bright quadruply imaged quasar B1422+231, B2045+265 (Fassnacht et al. 1999a) or B1608+656 (Fassnacht et al. 1999b) turn out to be very difficult to model. For all these systems, either the lens model is wrong or it could be that the structure of the radio source is more complex than originally assumed such that the estimated flux ratios cannot be compared with the observed ones. For the latter, the lens might consist of several sub-systems. Koopmans and de Bruyn (2000) also observed flux variations in the doubly imaged radio source B1600+434 at 8.5 and $4.9 \mathrm{GHz}$, which might be due to microlensing of very compact superluminal jets.

Up to now, the JVAS-CLASS survey constitutes the largest survey that has been carried out to identify gravitational lenses. However, it is not yet complete in a statistical sense since the most interesting gravitational lens candidates have probably been looked at first. It is probable that other candidates consisting of double sources, or unusual configurations for the multiple images, will either not show up in the optical or that the (sometimes very distant or possibly dark, etc.) lens will remain undetected. It is therefore not clear to which degree this radio survey will be complete. In addition, the parent population of the radio sources ought to be well defined as a function of redshift and here also, it is easy to foresee major difficulties in detecting their optical counterpart, and thus measuring their redshift, in a complete way. Finally, the source redshifts of the CLASS lensed sources turn out to be smaller than those found in optical surveys, rendering the JVAS/CLASS sample less sensitive to the cosmological parameters (Helbig et al. 1999).

\section{A harvest of multiply imaged QSOs}

Past and ongoing surveys discussed in Section 3 have led altogether to the discovery of more than 50 multiply imaged sources. Their general properties as 
well as some more specific characteristics are summarized in Table 3 . This Table contains the most updated observational status of multiply imaged QSOs at the time of writing this review. A version of this Table is also available through Internet via the URL: http://vela.astro.ulg.ac.be/grav_lens and will be regularly updated. Many lens detections and magnitude measurements are due to the CfA-Arizona Space Telescope LEns Survey (CASTLES), which consists of an ongoing HST direct imaging program in the $\mathrm{H}$, I and $\mathrm{V}$ bands, of all the known gravitational lenses as well as published candidates (see the URL: http://cfawww.harvard.edu/castles and Muñoz et al. 1999a).

Table 3. Individual observational properties of the multiply imaged QSOs: status in March 2000. More details on the astrometry and photometry of the individual lensed images can be found at the CASTLES Web site as well as at URL: http://vela.astro.ulg.ac.be/grav_lens

Table 3 contains the following information:

Column 1: the standard source name or any other frequently used designation,

Column 2: the survey(s) in which the gravitational lens system has been identified (see text),

Column 3: the number $\mathrm{N}$ of lensed images and/or other image characteristic(s),

Column 4: typical angular separation(s) ( $\Delta \theta$ in arcsec) between the multiple lensed images or diameter of the ring, etc.,

Column 5: spectral range(s) (O[ptical], R[adio] or X[-ray]) in which the lensed images have been detected,

Column 6: spectroscopic redshift $z_{s}$ of the source,

Column 7: spectroscopic redshift $z_{l}$ of the lens,

Column 8: physical source type (cf. quasar, jet, ...),

Column 9: physical lens type (cf. elliptical or spiral galaxy, ...),

Column 10: integrated magnitude(s) of all lensed source images. If the source and lens images cannot be resolved, we list the total magnitude of the lensed and lens images (cf. $\left.R_{t o t}, \ldots\right)$,

Column 11: magnitude(s) of the lens. If the lens and source images cannot be resolved, we list the total magnitude of the lens and lensed images (cf. $\left.R_{t o t}, \ldots\right)$,

Column 12: if known, an estimate of the lens mass times the Hubble constant expressed in unit of $100 \mathrm{~km} / \mathrm{sec} / \mathrm{Mpc}$. The mass is given in solar mass unit,

Column 13: if known, an estimate of the lens mass to light ratio divided by the Hubble constant expressed in unit of $100 \mathrm{~km} / \mathrm{sec} / \mathrm{Mpc}$. The spectral band used for the observed light is indicated between parentheses, 
Column 14: additional observed characteristics of the gravitational lens system (cf. time delay(s), detection of microlensing or differential reddening between the lensed images),

Column 15: astrophysical or cosmological application that has been made for the gravitational lens system (cf. estimate of $H_{0}$, of the size of intervening gas clouds located along the line-of-sight, of the deflector extinction law ...),

Column 16: condensed reference(s) of the main papers reporting the discovery of the gravitational lens system (first reference) as well as additional information listed in the present table. A question mark (?) appearing in the different columns indicates an approximate or doubtful value. See the precise references at the end of this paper (from PospieszalskaSurdej et al. 2000)

\section{Contributions of gravitational lensing to cosmology and astrophysics}

In this Section, we summarize the most important and most recent results achieved by the numerous studies of gravitational lensing in the quasar population. It is intended as a quantitative answer to the qualitative motivations exposed in Section 2.

\subsection{The cosmological parameters $\Omega_{o}$ and $\lambda_{o}$}

As explained in Section 2, gravitational lensing yields a natural upper limit on the value of the cosmological constant $\lambda_{o}$.

The first constraints on $\lambda_{o}$ have been obtained for flat cosmologies, with simple lens models and relatively small optical samples $\left(\lambda_{o}<0.8\right.$ at $90 \%$ confidence level (CL) in a sample of 653 HLQs, Kochanek 1993b; $\lambda_{o}<0.7$ at $95 \%$ CL with 494 HLQs, Maoz \& Rix 1993). The constraint was then progressively reinforced as the size of the observed sample was growing $\left(\lambda_{o}<0.66\right.$ at $95 \%$ CL with 862 HLQs, Kochanek 1996; $\lambda_{o}<0.55$ at the $90 \%$ CL with 1178 HLQs, Claeskens et al. 1996b, Claeskens 1999). Including in the likelihood analysis of 1164 HLQs the lens redshift or the lens (non)detection, Claeskens (1999) obtains $\lambda_{o}<0.50$ at the $95 \%$ CL: the redshift and magnitude distributions of the lenses are also compatible with a low value of $\lambda_{o}$. In all cases, the Einstein - de Sitter Universe $\left(\Omega_{o}=1, \lambda_{o}=0\right)$ is acceptable and even favoured by GL statistics (see Fig. 15).

However, a positive value of $\lambda_{o} \sim 0.7$ has been suggested from the analysis of high-z supernovae (Perlmutter et al. 1999, Riess et al. 1998). If it is confirmed, this could mean that the observed number of lenses in HLQ samples is too low, either because the galaxy luminosity function is not correct (Chiba \& Yoshii 1999) or because of extinction by dust in the lens (Malhotra 1997). Although Kochanek (1996) and Claeskens (1999) show that extinction in the lens is probably not sufficient to reconcile the results with a cosmological model dominated by $\lambda_{o}$, extinction by dust remains a critical issue as it also affects the completeness of the optical QSO samples. Let us note here that the presence of dust could also mimic the effect of a positive $\lambda_{o}$ on the supernova magnitudes in a $\lambda_{o}=0$ Universe (Aguirre 1999, Aguirre \& Haiman 1999). 


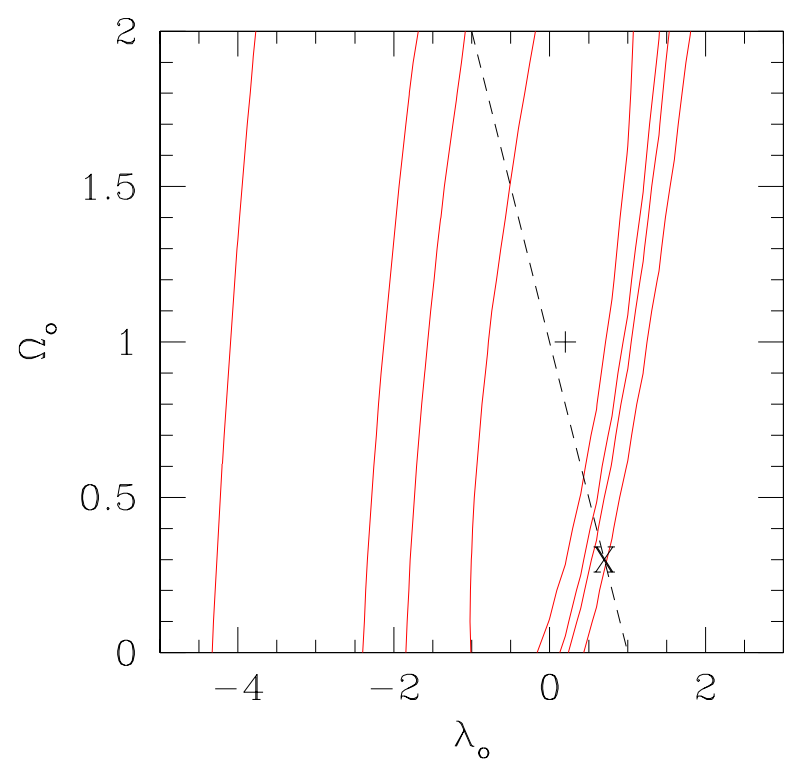

Fig. 15. Likelihood contours in the plane $\lambda_{o}-\Omega_{o}$ as derived from the observations of 1164 HLQs in the merged optical sample (see Table 2). The successive concentric contours enclose the true values of the parameters with a probability of $68.3,90,95.4$ and $99.7 \%$ respectively. The most likely value corresponds to the symbol "+" while the "X" symbol marks the corresponding result obtained from the analysis of the Supernova distances (Riess et al. 1998). The dashed line represents the locus of flat Universes $\left(\Omega_{o}+\lambda_{o}=1\right)$. Note the lack of constraint on $\Omega_{o}$.

Searches for multiple images in uniform radio surveys are not affected by the issue of extinction effects. Although the source population entering the computation of the amplification bias is poorly known, the statistics have been performed for the radio JVAS survey of 2500 flat-radio spectrum sources (see Section 3.2) by Falco et al. $1998\left(\lambda_{\circ}<0.73\right.$ at $95 \%$ CL) and by Helbig et al. $1999\left(\lambda_{\circ}<0.84\right.$ at $95 \% \mathrm{CL})$. The better agreement between those results and the supernovae results allow a joint analysis which yields $\lambda_{o}-\Omega_{o}<0.6$, i.e. $\lambda_{o}<0.8$ at $95 \% \mathrm{CL}$ in a flat cosmology (Helbig 1999). Additional results from the CLASS survey (see Section 3.2) and a better knowledge of the parent radio source population are mandatory (see Cooray 1999).

\subsection{Cosmological density of dark compact objects}

A wide panorama on existing constraints or theoretical predictions on putative cosmological populations of compact objects may be found with associated references in Dalcanton et al. (1994). Hereafter, we give more details from the contributions of lensing studies.

In 1982, Canizares got the first constraint on the cosmological density $\Omega_{L}$ of dark compact objects from the small observed variations in the equivalent width of broad emission lines in the spectrum of quasars: $\Omega_{L}<1$ for dark 


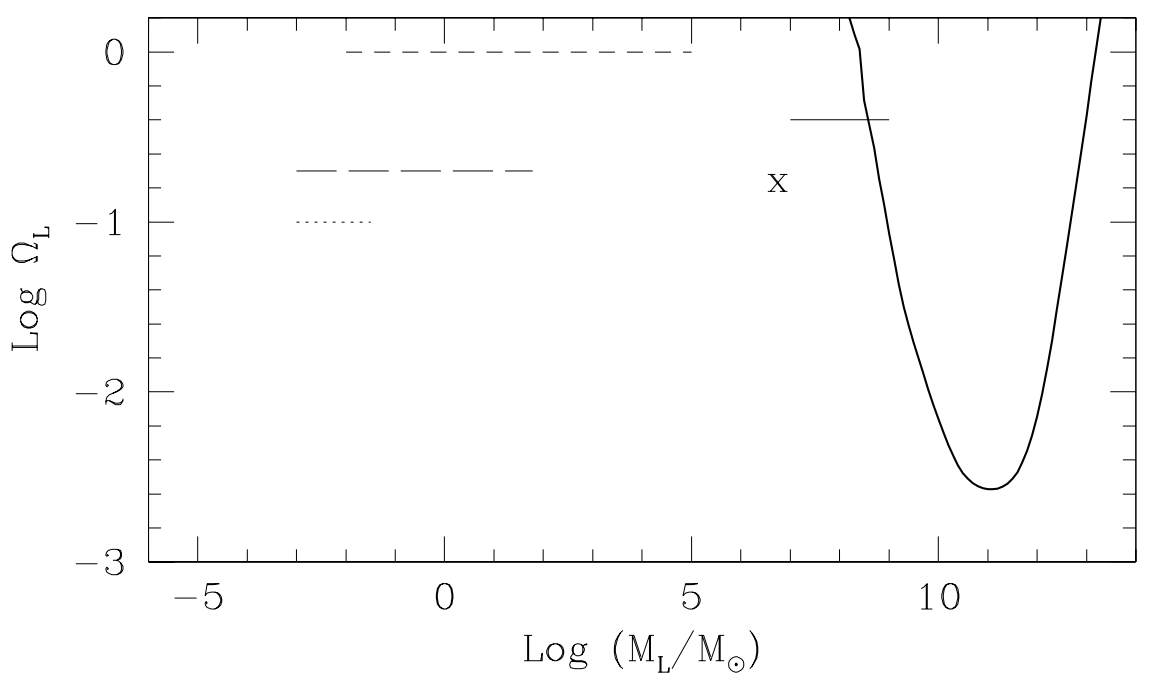

Fig. 16. Current constraints from gravitational lensing on the cosmolgical density of dark compact objects (in unit of the critical density $\Omega_{c}$ ) as a function of their mass. Thick full line: Claeskens 1999 (99.7\% CL); straight full line: Kassiola et al. 1991 (99.7\% CL); short dashed line: Canizares 1982; dotted line: Schneider 1993 ( 97\% CL); long dashed line: Dalcanton et al. 1994; "X": Marani et al. 1999 (90\% CL).

compact objects with $10^{-2} \leq \mathrm{M} / \mathrm{M}_{\odot} \leq 10^{5}$. Further constraints were provided by counting multiply imaged sources in the VLA lens survey (resp. in the first HLQ surveys) by Hewitt (1986) (resp. Nemiroff 1991). They were improved respectively by Kassiola et al. (1991), who took advantage of the higher angular resolution of the VLBI, and by Surdej et al. (1993a) who made use of a larger HLQ sample and included the amplification bias.

Fig. 16 summarizes the current best constraints on $\Omega_{L}$ in the mass range $10^{-3}-10^{14} \mathrm{M}_{\odot}$.

In the $10^{-3}-10^{5} \mathrm{M}_{\odot}$ mass range, i.e. in the microlensing domain (see Fig. 12), Canizares' results have partially been improved by Dalcanton et al. (1994) and Schneider (1993). The former also uses the equivalent width variations of emission lines while the latter studies the statistical variability of the quasar flux in a sample. Note that this variability can only be probed for a limited mass range. The lower limit $\left(\sim 10^{-4} \mathrm{M}_{\odot}\right)$ is due to the finite size of the QSO continuum emission region; the upper limit depends on our ability to record long microlensing events (about 10 years for a $1 \mathrm{M}_{\odot}$ cosmological object). Those results already preclude the existence of a cosmological population of stellar black holes or dwarf stars able to stop the expansion of the Universe.

A mass of $10^{6} \mathrm{M}_{\odot}$ corresponds to the Jeans' mass at recombination in Cold Dark Matter (CDM) models and is within the mass range of pregalactic objects predicted by several models (Carr \& Rees 1984, Gnedin \& Ostriker 1992). The cosmological density of such dark compact objects is smaller than 0.15 at $90 \%$ CL (Marani et al. 1999) because no echoes due to gravitational time delays were observed in Gamma-Ray bursts. Stronger constraints in this interesting mass 
range could be obtained with VLBA observations of radio sources, but this is not possible until the luminosity function of the source population is known.

Finally, the strongest constraint is $\Omega_{L}<0.003$ for $M_{L} \simeq 10^{11} \mathrm{M}_{\odot}$ at $99.7 \%$ CL (Claeskens 1999). It results from the search for multiply imaged HLQs with no apparent lens in a sample of 1164 objects. This means that massive objects with $10^{11} \mathrm{M}_{\odot}$ are nearly always in the form of visible galaxies and not in the form of dark haloes, as predicted in the CDM model by Navarro et al. (1996).

\subsection{The Hubble constant $H_{O}$}

Much progress has taken place during the past 15 years on the long way from theory to practice in determining $H_{o}$ with lensing studies.

First of all, the number of known lenses has continuously been increasing (see Table 3).

Secondly, much effort has been made in order to reduce the uncertainties in the different factors entering Eq. (40) which relates $H_{o}$ to observed quantities. So, image positions are well defined by radio or HST observations (e.g. CASTLES), the lens models are better constrained owing to velocity dispersion measurements of the main lens (e.g. Falco et al. 1997a, Tonry and Franx 1999 for Q0957+561) and to the detection of extended images of some QSO host galaxies e.g. for MG0414+0534 (Falco et al. 1997b, Angonin et al. 1999), Q0957+561 (Falco et al. 1997a, Bernstein et al. 1997), BRI0952-0115 and HE1104-1805 (Lehár et al. 1999), PG1115+080 (Impey et al. 1998), H1413+117 (Alloin et al. 1998), CTQ414 (Morgan et al. 1999) and Q2237+0305 (Mediavilla et al. 1998).

Thirdly, the importance of sustained photometric monitoring has been recognized in order to precisely determine time delays. In the case of Q0957+561, the long standing debate between the "long" (Press et al. 1992a,b) and the "short" (Vanderriest et al. 1989, Schild 1990, Schild and Thomson 1995, 1997, Pelt et al. 1996) observed time delays has finally been closed with $\Delta t_{12}=417 \pm 3$ days (Kundić at al. 1997a, confirmed by Haarsma et al. 1999), definitively in favor of the short time delay. Today, 6 other lens systems have measured time delays (see Table 3): B0218+357 (Biggs et al. 1999), HE1104-1805 (Wisotzki et al. 1998), PG1115+080 (Schechter et al. 1997, Barkana 1997), B1600+434 (Koopmans et al. 2000), B1608+656 (Fassnacht et al. 1999b) and PKS1830-211 (Lovell et al. 1998).

Although some complementary information has been obtained on the deflector from observations (such as the velocity dispersion of the lens or the convergence term from weak lensing studies), the lens model cannot be fully constrained from the observations and it becomes now the dominant source of uncertainty on the derived value of $H_{o}$, especially through its radial logarithmic slope. Despite the fact that the lens of Q0957+561 is complex (presence of a cluster), it is probably still the best constrained system. Using their model, Falco et al. (1997a) find $H_{o}=62-67 \pm 7 \mathrm{~km} / \mathrm{s} / \mathrm{Mpc}$ at $2 \sigma$, and Kundić et al. (1997a) derive $H_{o}=64 \pm 13 \mathrm{~km} / \mathrm{s} / \mathrm{Mpc}$ at $2 \sigma$ on the basis of the Grogin and Narayan (1996) lens model. However, allowing for more general models (including ellipticity of the lens), Bernstein and Fischer (1999) find larger uncertainties: $H_{o}=77_{-24}^{+29} \mathrm{~km} / \mathrm{s} / \mathrm{Mpc}$ at $2 \sigma$. A non exhaustive list of values for $H_{o}$ derived hitherto from Q0957+561 and other multiply imaged QSOs is presented in Table 4 . 
Table 4. Determinations of $H_{o}$ from gravitational lensing. Unless stated otherwise, error bars are $2 \sigma$ uncertainties

\begin{tabular}{lcl}
\hline \hline Lens & $H_{o}(\mathrm{~km} / \mathrm{s} / \mathrm{Mpc})$ & References \\
\hline B0218+357 & $69_{-19}^{+13}$ & Biggs et al. (1999) \\
\hline Q0957+561 & $62-67 \pm 7$ & Falco et al. (1997a) \\
& $64 \pm 13$ & Kundić et al. (1997a) \\
& $72 \pm 7(1 \sigma)$ & Tonry and Franx (1999) \\
& $55_{-14}^{+15}$ & Chae (1999) \\
& $77_{-24}^{+29}$ & Bernstein and Fischer (1999) \\
& $53_{-7}^{+10}$ & Courbin et al. (1997) \\
\hline PG1115+080 & $52 \pm 14$ & Kundić et al. (1997b) \\
& $44 \pm 4(1 \sigma)$ & Impey et al. (1998) \\
\hline B1608+656 & $59_{-7}^{+8} \pm 15$ & Fassnacht et al. (1999b) \\
\hline PKS1830-211 & $65_{-9}^{+15}(1 \sigma)$ & Lidman et al. (1999) \\
\hline
\end{tabular}

\subsection{The lensing galaxies}

Lensing galaxies are difficult to observe since they are expected to be faint and extended high redshift objects, located between bright and angularly very close $\left(\sim 1^{\prime \prime}\right)$ multiple images of the background quasars. However, mainly thanks to the high angular resolution of the HST and to the use of powerful image analysis techniques (such as Point Spread Function subtraction, profile fitting and deconvolution), the lens has been detected for about $91 \%$ of the multiply imaged QSOs (see Table 3). Even, the morphological type could be obtained for $61 \%$ of them. It is apparent that the most common lenses are isolated elliptical galaxies, but spiral galaxies are also observed (e.g. B0218+357, $\mathrm{B} 1600+434, \mathrm{~B} 1608+656, \ldots)$. The main lens can also be immersed in a galaxy cluster (e.g. Q0957+561, B1422+231,...) or be composed of more than one galaxy (e.g. B1127+385, MG2016+112,...). The lens spectrocopic redshifts are still more difficult to measure: they are presently known for only 27 lenses, i.e. $52 \%$. The median value is $z_{D}=0.47$, as expected from Fig. 6 . In some cases, the lens redshift may possibly be derived from an association with that of a spectroscopic absorption line system seen in the spectra of the lensed images or by studies of their differential reddening (see Section 5.5).

Multiply imaged QSOs provide unique information on the mass distribution of these high redshift lensing galaxies.

First, the predominance of ellipticals as gravitational lenses over spirals was expected from their larger mass, producing a larger cross-section for lensing and a wider mean angular separation between the lensed images which are thus easier to detect (see Eqs. (13), (38) and Fukugita and Turner 1991). This is confirmed by statistical studies of the angular separations of the multiple images present in QSO samples (Kochanek 1993b, Kochanek 1996, Falco et al. 1998, Claeskens 1999). However, the proportion of spirals is higher in the CLASS survey (see Table 3 and Jackson et al. 1998a). Möller and Blain (1998) claim that the latter have an increased lensing cross-section with a higher related amplification bias due to the inclination of the disk and that spiral lenses would only show up when the survey is not sensitive to dust as in the radio, but this is contradicted 
by the results of Keeton and Kochanek (1998). Gravitational lensing by spiral galaxies should allow us to better study the relative contributions of their disk, bulge and surrounding halo.

Second, the relative number of quadruply imaged and doubly imaged QSOs in optical samples also seems to be explained by a reasonable ellipticity of the lensing potential (Claeskens 1999), although the comparison with the ellipticity of the light distribution is dangerous (Keeton, Kochanek and Falco 1998). The absence of observed cosmic mirages with 3 images has soon been recognized as a signature of nearly singular potentials (Wallington and Narayan 1993, Kochanek 1996). This is in agreement with HST observations of local elliptical galaxies by Gebhardt et al. (1996). It remains unclear however why the proportion of quadruply/doubly imaged radio sources is so high in the CLASS survey (Jackson et al. 1998a,b), but this could be due to a pure observational selection bias.

Third, the statistical studies also suggest that the observations are better reproduced by lensing isothermal dark matter halos rather than by constant $M / L$ lens models (Maoz and Rix 1993, Kochanek 1996). However, for individual lenses, simple models are not always able to adequately fit the image positions and flux ratios. The introduction of an additional shear term may help but its origin cannot always be explained by the perturbation of galaxies close to the main lens (Keeton et al. 1997).

Finally, it is interesting to combine the mass of the lens estimated from model fitting ${ }^{14}$, with the photometric data. The resulting $M / L$ ratio is found to be in the range 10-20 in the $B$ band (Keeton, Kochanek and Falco 1998, see also Jackson et al. 1998b). Kochanek et al. (1999) show that most gravitational lenses are compatible with elliptical galaxies lying on the same passively evolving fundamental plane as the elliptical galaxies residing in clusters.

\subsection{Dust extinction in the lens galaxies}

In order to reconcile the observed number of multiply imaged QSOs in the optical samples presented above with the expected one in a Universe with $\lambda_{o} \simeq 1$, the mean extinction in the lens galaxies ought to be $A_{V} \simeq 2$ mag (Kochanek 1996, Claeskens 1999). A smaller value, corresponding to about $0.5 \mathrm{mag}$ in the $B$ band, seems to be needed to improve the fit of the merged (optical + radio) samples and to retrieve the constraints on $\lambda_{o}$ derived from radio samples (Falco et al. 1998). This is at the level of the observed extinction in local external galaxies (see e.g. Peletier 1995, Van Dokkum \& Franx 1995). Malhotra et al. (1997) made the first attempt to estimate the extinction at higher redshift from the analysis of the colours of a sample of lensed radio sources and lensed optically selected ones. But one cannot exclude intrinsic dispersion in the colours. Only differential reddening can directly be measured. From a sample of 23 lens galaxies, Falco et al. (1999) find a mean value of $\Delta E_{B-V}=0.05^{15}$. They tentatively come to the conclusion that the derived total extinction in the lens accounts for the different

\footnotetext{
14 The mass between the images is a robust, model independent estimate (see Eqs. (14) and (15)).

15 This weak differential extinction confirms that no multiply imaged QSO is missing in the existing HLQ optical samples (see Claeskens and Surdej 1998).
} 
values of $\lambda_{o}$ obtained from statistical studies of optically selected samples and of radio-source samples.

Using existing heterogeneous photometric observations, Jean and Surdej (1998) applied their method to simultaneously fit the $R_{V}$ extinction parameter and the redshift $z_{l}$ of the lensing galaxies responsible for the quadruply imaged QSOs H1413+117 and MG0414+0534. They found in both cases a value of $z_{l} \simeq 1.15$. The redshift of the latter galaxy has been spectroscopically confirmed to be $z_{l}=0.96$ (Tonry and Kochanek 1999). "Dust redshift" estimates for other gravitational lenses have been performed by Falco et al. (1999). Better photometry and high quality spectroscopic data of selected lenses should very soon provide clues on their extinction curves.

\subsection{Size of QSOs}

Analysis of the gravitational microlensing high amplification event reported for Q2237+0305A by Irwin et al. (1989) has led Wyithe et al. (1999) to the conclusion that upper and lower limits (at 99\% CL) on the intrinsic size of the $R$-band continuum emitting region are $610^{15}$ and $210^{13} \mathrm{~cm}$, respectively (see also Racine 1992 and Lewis et al. 1998 for previous estimates). A value of $10^{15}-10^{16} \mathrm{~cm}$ for the size of the quasar Q0957+561 has similarly been proposed by Pelt et al. (1998); see also Schmidt and Wambsganss 1998.

Let us mention here that the resulting amplification due to lensing galaxy has helped Alloin et al. $(1998,1997)$ and Yun et al. (1997) to resolve CO(7-6) extended emission at high redshift from the lensed quasar H1413+117; Barvainis et al. (1998) similarly detected CO(3-2) emission from MG0414+0534.

\subsection{Size of intergalactic clouds}

The large number of coincident absorption lines found in the spectra of the multiply imaged QSOs UM673 A\&B (Smette et al. 1992) and HE1104-1805 (Smette et al. 1995) implies that most of the intervening clouds extend over both lines of sight. A lower limit on the size of the clouds was derived to be $6 h^{-1} \mathrm{kpc}$ and $50 h^{-1} \mathrm{kpc}$ respectively $\left(h=H_{o} / 100\right)$, in agreement with even larger estimates of about $300 h^{-1} \mathrm{kpc}$ recently found from spectroscopic studies of QSO pairs (D'Odorico et al. 1998).

\subsection{The distorted apparent Universe}

Gravitational lensing also distorts our view of the distant Universe, although not as strongly as first suggested by Barnothy and Barnothy (1968). Indeed, the amplification bias, which helps in discovering new mirages (see Section 1.8.2), can also affect the astrophysical properties inferred from flux limited samples. Here, we briefly discuss three examples related to flux limited QSO samples.

First, the apparent QSO luminosity function could be modified and a part of its apparent evolution with redshift could be due to gravitational lensing, as first pointed out by Turner (1980). Schneider (1987) showed that the effect could indeed be important provided the sources are as small as derived from their 
variability, a small fraction of the Universe is in the form of compact objects and the intrinsic luminosity function is sufficiently steep. The effect should thus be more important at the bright end. Directly evaluating the amplification bias from the observed luminosity functions of lensed and unlensed quasars has not been possible yet because of the lack of large homogeneous surveys. This should become feasible when surveys like the SDSS are completed in the near future. However, the analysis of the bright end of the QSO luminosity function in the Hamburg/ESO survey already shows that it is steeper at higher redshift. This is the opposite of what is expected since gravitational lensing being more frequent at higher redshift, the amplification bias should flatten the bright end of the apparent luminosity function (Wisotzki 2000).

Second, the amplification bias should create an "artificial" correlation at very small angular separations (few arcsec) between high redshift background QSOs and foreground visible galaxies in flux limited samples, even if the former are not multiply imaged (weak lensing). However, the number density of the sources is also diluted by gravitational lensing and Narayan (1989), Kayser and Tribble (1991), Claeskens and Surdej (1998) and others showed that the resulting expected overdensity of galaxies in the angular vicinity of QSOs is very low and cannot reproduce the highest reported ones. The latter authors also claim that the comparison is hampered by small number statistics and possible selection biases and that about 1500 HLQs should be observed down to a limiting magitude of $R_{\text {lim }} \sim 23$ before a definite conclusion can be drawn. Positive angular correlations between high redshift QSOs and foreground galaxies are also observed on larger scales (up to one degree) and may be used to constrain large scale structures (see e.g. Williams and Irwin 1998 and references therein).

Finally, as a last example, let us mention that gravitational lensing might affect the determination of the cosmological density $\Omega_{\mathrm{HI}}$ of neutral hydrogen based on Damped Ly $\alpha$ Absorptions (DLAs) surveys in quasar spectra (Bartelmann and Loeb 1996; Smette, Claeskens and Surdej 1997). Indeed, lensing by the halo of the associated spiral galaxies could cause the light rays from the background QSOs to avoid the densest central parts of the disk, while the amplification bias increases the chance that a QSO with a DLA be included in a flux limited sample. The competition between these two effects results in a slight overestimation of $\Omega_{\mathrm{HI}}$ in existing high redshift surveys but the latter could be more significant at lower redshift in bright QSO samples (Smette et al. 1997), although the influence of dust extinction could balance that of the amplification bias (Bartelmann and Loeb 1998).

\section{Conclusions}

The first cosmic mirage was found by chance. This happened in 1979, with the discovery of the doubly imaged radio-loud quasar Q0957+561 (Walsh et al. 1979). Hunting the cosmic illusions in the distant Universe then became a sort of favorite sport for some adventurous observational cosmologists and the known number of multiply imaged QSOs slowly increased during the 80's, reaching 7 in 1990 (Surdej and Soucail 1993). Later on, the scientific interest in this phenomenon being widely recognized, finding gravitational lenses became a popular sport: several independent teams initiated surveys among known HLQs or radio sources. At the IAU meeting of Melbourne in mid 1995, 24 systems were 
known, among which 16 were classified as certain (Keeton and Kochanek 1996). In early 2000 , more than 50 multiply imaged QSOs or radio sources are known and $91 \%$ of the lenses are detected (see Table 3). A further exponential increase must be expected since the period of "artisanal" astronomical observations is being progressively replaced by the "industrial" era of automated surveys. At the same time, the observations are going to be deeper and deeper. They will reach a huge population of faint and extremely distant sources, and the more distant the source, the larger the chance to get a gravitational lens along the same line-of-sight. As a consequence, Barkana et al. (2000) estimate that the Next Generation Space Telescope (NGST) should detect several lensed objects in each field of view... What was gold for the astronomers of the 80's could transform into lead for the next generation of astronomers, who will first need to remove lensing artefacts before discovering the astrophysical properties of the primeval objects...

In this review, we tried to illustrate the numerous potential applications of strong lensing and to summarize some interesting results already achieved. Automated QSO surveys (SDSS, 2dF) and important radio surveys like CLASS will provide large, complete and homogeneous samples of distant sources, free of uncontrolled selection bias. They will undoubtedly improve the statistical studies based on gravitational lensing; the studies of the optical QSO luminosity function and of the properties of the lensing galaxy population (extinction, $M / L, \ldots$ ) will also necessarily benefit from this. Newly discovered lenses will also increase our opportunity to determine $H_{o}$. Finally, important discoveries will surely concern the large scale structures at moderate redshifts, thanks to the study of weak lensing on the myriads of very high redshift background sources which are going to be resolved with the next generation of large telescopes. But this is another story...

Acknowledgement. We are very grateful to Prof. L. Woltjer for having invited us to write the present review on Gravitational Lensing in Quasar Samples and for his valuable suggestions on the first manuscript. Our research was supported in part by contract P4/05 "Pôle d'Attraction Interuniversitaire" (OSTC, Belgium), by PRODEX (Gravitational lens studies with HST), by the "Fonds National de la Recherche Scientifique" (Belgium) and by contract ARC94/99-178 "Action de Recherche Concertée de la Communauté Française de Belgique". Our thanks also go to Jean-Pierre Swings and to Valery Zhdanov for reading the manuscript and to Anna Pospieszalska for her help with the tables and with the Gravitational Lens Bibliography.

\section{References}

Agol, E., Krolik, J., 1999 ApJ 524, 49

Aguirre, A.N., 1999 ApJ 512, L19

Aguirre, A.N., Haiman Z., 1999 ApJ, submitted astro-ph/9907039

Albrow, M., Beaulieu, J.-P., Birch, P. et al., 1998 ApJ 509, 687

Alloin, D., Guilloteau, S., Barvainis, R. et al. 1997 A\&A 321, 24

Alloin, D., Mellier, Y., Kneib, J.-P., 1998 in Proceedings "Molecular Lines at High Redshift", eds. C. Arilli, S. Radford, K. Menten, Greenbank

Altieri, B., Metcalfe, L., Kneib, J.P. et al. , 1999 A\&A 343, L65

Angonin-Willaime, M.-C., Vanderriest, C., Courbin, F. et al., 1999 A\&A 347, 434

Augusto, P., Wilkinson, P.N., Browne, I.V.A.: 1996, IAU Symp. No.173, (Melbourne) "Astrophysical Applications of Gravitational Lensing", p.399, [AUG96]

Bade, N., Siebert, J., Lopez, S. et al., 1997 A\&A 317, L13, [BAD97.1] 
Bahcall, J.N., Hartig, G.F., Jannuzi, B.T. et al., 1992 ApJ 400, L51, [BAH92.1]

Barkana, R., 1997 ApJ 489, 21, [BAR97.1]

Barkana, R., Blandford, R., Hogg, D., 1999 ApJ 513, L91, [BAR99.1]

Barkana, R., Hogg, D.W., Loeb, A. et al. 2000 Proceedings of the Colloquium "Gravitational Lensing: Recent Progress and Future Goals", Boston 1999, editors T. Brainerd \& C.S. Kochanek, in press; astro-ph/0001325

Barnothy, J.M., Barnothy, M.F., 1968 Science 162, 348

Bartelmann, M., Loeb, A., 1996 ApJ 457, 529

Bartelmann, M., Loeb, A., 1998 ApJ 503, 48

Bartelmann, M., Schneider, P., 1999, astro-ph/9912508

Barvainis, R., Alloin, D., Guilloteau, S., Antonucci, R., 1998 ApJ 492, L13

Barvainis, R., Antonucci, R., Helou, G. 1999 AJ 118, 645

Bernstein, G., Fischer, Ph., Tyson, J.A., Rhee, G., 1997 ApJ 483, L79

Bernstein, G., Fischer, Ph., 1999 AJ 118, 14

Bézecourt, J., Soucail, G., Ellis, R. S., Kneib, J.-P, 1999 A\&A 351, 433

Biggs, A.D., Browne, I.W.A., Helbig, P. et al., 1999 MNRAS 304, 349, [BIG99.1]

Blain, A.W., 1998 MNRAS 297, 511

Blandford, R., Narayan, R., 1986 ApJ 310, 568

Blandford, R., Kochanek, C.S., 1987 ApJ 321, 658

Boyle, B.J., Shanks, T., Peterson, B.A., 1988 MNRAS 235, 935

Bunker, A.J., Moustakas, L.A., Davis, M., 2000 ApJ in press, astro-ph/9909476

Burke, W.L., 1981, ApJ 244, L1

Burud, I., Courbin, F., Lidman, C. et al., 1998 ApJ 501, L5, [BUR98.1]

Canizares, C.R., 1982 ApJ 263, 508

Carr, B.J., Rees, M.J., 1984 MNRAS 206, 315

Chae, K.Y., 1999 ApJ 524, 582

Chang, K., Refsdal, S., 1979 Nature 282, 561

Chavushyan, V.H., Vlasyuk, V.V., Stepanian, J.A., Erastova, L.K., 1997 A\&A 318, L67, [CHA97.1]

Chiba, M., Yoshii, Y., 1999 ApJ 510, 42

Chwolson, O., 1924, Astr. Nachrichten 221, 329

Claeskens, J.-F., Surdej, J., Remy, M., 1996a A\&A 305, L9, [CLA96.1]

Claeskens, J.-F., Jaunsen, A.O., Surdej, J., 1996b, Proc. IAU 173 “Astrophysical Applications of Gravitational Lensing”, p13- 20 (éditeurs C.S. Kochanek, J.N. Hewitt).

Claeskens, J.-F., Surdej, J., 1998 A\&A 335, 69

Claeskens, J.-F., 1999 Thèse de Doctorat, Soc. Roy. Sci. Liège 68, 1-305

Cooke, J.H., Kantowski, R., 1975 195, L11

Cooray, A.R., 1999 A\&A 342, 353

Courbin, F., Magain, P., Keeton, C.R., Kochanek, C.S. et al., 1997 A\&A 324, L1

Crampton D., McClure, R.D., Murray Fletcher, J., 1992 ApJ 392, 23

Crampton, D., Le Fèvre, O., Hammer, F., Lilly, S.J., 1996 A\&A 307, L53

Crampton, D., Schechter, P.L., Beuzit, J.-L., 1998 AJ 115, 1383, [CRA98.1]

Dalcanton, J.J., Canizares, C.R., Granados, A. et al., 1994 ApJ 424, 550

D’Odorico V., Cristiani S., D'Odorico S. et al. 1998 A\&A 339, 678

Einstein, A., 1936, Science 84, 506

Eisenhard, P.R., Armus, L., Hogg, D.W. et al., 1996 ApJ 461, 72, [EIS96.1]

Engels, D., Hagen, H.-J., Cordis, L. et al. 1998 A\&AS 128, 507

Falco, E.E., Gorenstein, M.V., Shapiro, I.I., 1985 ApJ 289, L1

Falco, E.E., Shapiro, I.I., Moustakas, L.A., Davis, M., 1997a ApJ 484, 70

Falco, E.E., Lehar, J., Shapiro, I., 1997b AJ 113, 540

Falco, E.E., Kochanek, C.S., Muñoz, J.A., 1998 ApJ 494, 47

Falco, E.E., Impey, C.D., Kochanek, C.S. et al., 1999 ApJ 523, 617

Fassnacht, D., Cohen, J.G., 1998 AJ, 115, 377, [FAS98.1]

Fassnacht, C.D., Blandford, R.D., Cohen, J.G. et al., 1999a AJ 117, 658, [FAS99.1]

Fassnacht, C.D., Pearson, T.J., Readhead A.C.S. et al., 1999b ApJ 527, 498

Fischer, P., Schade, D., Barientos, F., 1998 ApJ 503, L127, [FIS98.1]

Fort, B., Mellier, Y., 1994 AAR 5, 239

Franx, M., Illingworth, G.D., Kelson, D.D. et al., 1997 ApJ 486, L75 
Fukugita, M., Futamase, T., Kasai, M., 1990 MNRAS 246, 24p

Fukuguta, M., Turner, E.L., 1991 MNRAS 253, 99

Fukugita, M., Futamase, T., Kasai, M., Turner, E., 1992 ApJ 393, 3

Gebhardt, K., Richstone, D., Ajhar, E.A. et al. 1996 AJ 112, 105

Gnedin, N.Yu., Ostriker, J.P., 1992 ApJ 400, 1

Gorenstein, M.V., Cohen, N.L., Shapiro, I.I. et al. 1988a ApJ 334, 42

Gorenstein, M.V., Falco, E.E., Shapiro, I.I., 1988b ApJ 327, 693

Gregg, M.D., Wisotzki, L., Becker, R. et al. 2000 AJ, submitted, astro-ph/0002333, [GRE00.2]

Grogin, N.A., Narayan, R., 1996 ApJ 464, 92 (erratum, 473, 570)

Haarsma, D.B., Hewitt, J.N., Lehar, J., Burke, B.F., 1999 ApJ, 510, 64

Hagen, H.-J., Reimers, D., $2000 A \& A$, in press, [HAG00.1]

Hartwick, F.D.A, Schade, D., 1990 ARA\&A 28,437

Hattori, M., Kneib, J.-P., Makino, N., 1999 Prog. Theor. Phys. Supp. 133 and astroph/9905009

Helbig ,P., 1999 A\&A in press , astro-ph/9904311

Helbig, P., Marlow, D., Quast, R. et al. 1999 A\&AS 136, 297

Hewitt, A., Burbidge, G., 1987 ApJS 63, 1

Hewitt, J.N., 1986 Ph.D. thesis MIT

Hewitt, J.N., Turner, E.L., Schneider, D.P. et al., 1988 Nature 333, 537, [HEW88.1]

Hewitt, J.N., Turner, E.L., Lawrence, C.R. et al., 1992 AJ 104, 968, [HEW92.2]

Huchra, J., Gorenstein, M., Kent, S. et al., 1985 AJ 90, 691, [HUC85.1]

Ibata, R.A., Lewis, G.F., Irwin et al., 1999 AJ 118, 1922, [IBA99.1]

Impey, C., Falco, E., Kochanek, C. et al., 1998 ApJ 509, 551

Irwin, M.J., Webster, R.L., Hewett, P.C. et al. 1989 AJ 98, 1989

Irwin, M.J., Ibata, R.A., Lewis, G.F., Totten, E.J., 1998 ApJ 505, 529, [IRW98.1]

Jackson, N., De Bruyn, A.G., Myers, S. et al., 1995 MNRAS 274, L25, [JAC95.1]

Jackson, N., Nair, S., Browne, I., 1998a in "Observational Cosmology with the New Radio Survey", Astrophysics and Space Science Library, Vol. 226, Dordrecht: Kluwer Acadademic Publishers (1998) (editors: M. Bremer, N. Jackson, I. Pèrez-Fournon)

Jackson, N., Helbig, P., Browne, I. et al. 1998b A\&A 334, L33, [JAC98.2]

Jean, C., Surdej, J., 1998 A\&A 339, 729

Jaunsen, A.O., Jablonski, M., Pettersen, B.R., Stabell, R., 1995 A\&A 300, 323

Jaunsen, A.O., Hjorth, J., 1997 A\&A 317, L39

Kassiola, A., Kovner, I., Blandford, R.D., 1991 ApJ 381, 6

Kassiola, A., Kovner, I., 1993 ApJ 417, 450

Kayser, N., Tribble, P., 1991 ASP Conference Series, 21, 304

Kayser, R., Helbig, P., Schramm, T., 1997 A\&A 318, 680

Keeton, C.R., Kochanek, C.S., 1996 Proc. IAU 173 "Astrophysical Applications of Gravitational Lensing” , p419 (editors C.S. Kochanek \& J.N. Hewitt).

Keeton, C.R, Kochanek, C.S., Seljak, U. 1997 ApJ, 482, 604

Keeton, C.R., Kochanek, C.S., 1998, ApJ 495, 157

Keeton, C.R, Kochanek, C.S., Falco, E.E., 1998, ApJ 509, 561

King, L.J., Browne, I.W.A., Muxlow, T.W.B. et al. 1997 MNRAS 289, 450

King L.J., Jackson, N.J., Blandford, R.D. et al., 1998 MNRAS 295, L41, [KIN98.1]

King, L.J., Browne, I.W.A., Marlow, D.R. et al. 1999 MNRAS 307, 225

Kneib, J.-P., Alloin, D., 1998 A\&A 339, L65, [KNE98.2]

Kochanek, C.S., 1991 ApJ 379, 517

Kochanek, C.S., 1993a MNRAS 261, 453

Kochanek, C.S., 1993b ApJ 419, 12

Kochanek, C.S., Falco, E.E., Schild, R., 1995 ApJ 452, 109

Kochanek, C.S., 1996 ApJ, 466, 638

Kochanek, C.S., Falco, E.E., Schild, R. et al., 1997 ApJ 479, 678, [KOC97.1]

Kochanek, C.S., Falco, E.E., Impey, C.D. et al., 1999 ApJ submitted, astro-ph/9909018

Koopmans, L.V.E., de Bruyn, A.G., Jackson, N., 1998 MNRAS 295, 534, [KOO98.1]

Koopmans, L.V.E., de Bruyn, A.G., Marlow, D.R. et al.,1999 Monthly Notices Royal Astron. Soc. 303, 727, [KOO99.1]

Koopmans, L.V.E., de Bruyn, A.G., Xanthopoulos, E., Fassnacht, C.D., 2000 A\&A in press, astro-ph/0001533 
Koopmans, L.V.E, de Bruyn, A.G., 2000 in Perspectives in Radio Astronomy: Scientific Imperatives at $\mathrm{cm}$ and $\mathrm{m}$ Wavelengths (Dwingeloo:NFRA), EdS. M.P. van Haarlem \& J.M. van der Hulst and astro-ph/0001108,

Kormann, R., Schneider, P., Bartelmann, M., 1994a A\&A 284, 285

Kundić, T., Turner, E.L., Colley, W.N. et al., 1997a ApJ 482, 75

Kundić, T., Cohen, J.G., Blandford, R.D., Lubin, L.M., 1997b AJ 114, 507

Lacy, M., Rawlings, S., Serjeant, S., 1998 MNRAS 299, 1220, [LAC98.1]

Langston, G.I., Schneider, D.P., Conner, S. et al., 1989 AJ 97, 1283, [LAN89.1]

Larkin, J.E., Matthews, K., Lawrence, C.R. et al., 1994 ApJ 420, L9

Lawrence, C.R., Schneider, D.P., Schmidt, M. et al. 1984 Science 223, 46, [LAW84.1]

Lawrence, C. R., Bennett, C. L., Hewitt, J. N., 1986 ApJS 61, 105

Lawrence, C.R., Elston, R., Januzzi, B.T., Turner, E.L., 1995 AJ 110, 2570

Lehár, J., Langston, G.I., Silber, A., 1993 AJ 105, 847, [LEH93.1]

Lehár, J., Burke, B.F., Conner,S.R. et al., 1997 AJ 114, 48, [LEH97.1]

Lehár, J., Falco, E.E., Kochanek, C.S. et al. 1999 ApJ , submitted; astro-ph/9909072

Ledoux, C., Théodore, B., Petitjean, P. et al., 1998 A\&A 339, L77, [LED98.1]

Lewis, G.F., Irwin, M.J., Hewett, P.C., Foltz, C.B., 1998 MNRAS 295, 573

Lewis, G.F., Ibata, R.A.,1999 ApJ In press, http://xxx.lanl.gov/abs/astro-ph/9908175

Lidman, C., Courbin, F., Meylan, G. et al. 1999 ApJ 514, L57

Lopez, S., Wucknitz, O., Wisotzki, L., 1998 A\&A 339, L13, [LOP98.2]

Loveday, J., 1996 Conference Paper, Rencontres De Moriond Workshop

Lovell, J.E.J., Jauncey, D.L., Reynolds, J.E. et al., 1998 ApJ 508, L51, [LOV98.1]

Lubin, L.M., Fassnacht, C.D., Readhead, A.C.S. et al., 2000 AJ 119, 451, [LUB00.1]

Magain, P., Surdej, J., Swings, J.-P., Borgeest U. et al. 1988 Nature 334, 325, [MAG88.1]

Magain, P., Surdej, J., Vanderriest, C., Pirenne, B., Hutsemékers, D., 1992 A\&A 253, L13 (Erratum: 1993, A\&A 272, 383), [MAG92.1]

Malhotra, S., Rhoads, J.E., Turner, E.L., 1997, MNRAS 288, 138

Maoz, D., Bahcall, J.N., Schneider, D.P. et al., 1993 ApJ 409, 28

Maoz, D., Rix, H.-W., 1993 ApJ 416, 425

Marani, G.F., Nemiroff, R.J., Norris, J.P. et al., 1999 ApJ 512, L13

Marlow, D. R., Browne, I. W. A., Jackson, N., Wilkinson, P. N., 1999 MNRAS 305, 15, [MAR99.2]

Marlow, D.R., Myers, S.T., Rusin, D. et al., 1999 AJ 118, 654, [MAR99.3]

McLeod, B.A., Bernstein, G.M., Rieke, M.J., Weedman, D.W., 1998 AJ 115, 1377, [MCL98.1]

McMahon, R., Irwin, M., Hazard, C., Corrigan, R., 1992 Gemini 36, 1, [MCM92.1]

Mediavilla, E., Arribas, S., del Burgo, C. et al. 1998, ApJ 503, L27

Metcalfe, L., Altieri, B., McBreen, B. et al. 1999 in "The Universe as seen by ISO", eds.: P.Cox, M.F.Kessler, ESA Publication Division, ESTEC, Noordwijk, NL

Möller, O., Blain, A.W., 1998 MNRAS 299, 845

Morgan, N.D., Dressler, A., Maza, J. et al. 1999 AJ 118, 1444, [MOR99.1]

Muñoz, J.A., Falco, E.E., Kochanek, C.S., et al. 1999a Astrophys. Space Science, sp. issue: Proc. III Scientific Meeting of the SEA, editors: J. Gorgas \& J. Zamorano

Muñoz, J.A., Kochanek, C.S., Falco, E.E. 1999b ApJ 521 L17

Muñoz, J.A., Falco, E.E., Kochanek, C.S. et al., 1999c Proc. ASP Conf. Ser. "Gravitational lensing: Recent Progress and Future Goals", eds. Brainerd, T., Kochanek, C.S., [MUN99]

Myers, S.T., Fassnacht, C.D., Djorgovski, S.G. et al., 1995 ApJ 447, L5, [MYE95.1]

Myers, S. T., Rusin, D., Fassnacht, C. D. et al. 1999 AJ 117, 2565, [MYE99.1]

Myers et al. 2000 in preparation

Nadeau, D., Yee, H.K.C., Forrest, W.J. et al. 1991, Astrophys. J. 376, 430

Narayan, R., 1989 ApJ 339, L53

Narayan, R., Bartelmann, M., 1996 Lecture on gravitational lensing and astro-ph/9606001

Navarro, J.F., Frenk, C.S., White, S.D.M., 1996 ApJ 462, 563

Nemiroff, R.J., 1988 ApJ 341, 579

Nemiroff, R.J., 1991 Phys. Rev. Lett. 66, 538

Paczyński, B., 1996, ARAA 34, 419

Patnaik, A.R., Browne, I.W.A., Walsh, D. et al., 1992 MNRAS 259, 1p, [PAT92.2]

Patnaik, A., Browne, I., King, L. et al., 1993 Proc. Sub-Arcsecond Radio Astronomy, 1992, Manchester, p.137, [PAT93.1] 
Patnaik, A.R., Browne, I.W.A., King, L.J. et al., 1993 MNRAS 261, 435, [PAT93.2]

Peebles, P.J.E., 1993 "Principle of Physical Cosmology", Princeton

Peletier, R.F., Valentijn, E.A., Moorwood, A.F.M. et al. 1995 A\&A 300, L1

Pello, R., Kneib, J.-P., Le Borgne, J.-F. et al. 1999 in "Clustering at High Redshift" Conference, June 29 to July 2, 1999, Marseille (France) and astro-ph/9910467

Pelt, J., Kayser, R., Refsdal, S., Schramm, T., 1996 A\&A 305, 97

Pelt, J., Schild, R., Refsdal, S., Stabell, R., 1998 A\&A 336, 829

Perlmutter, S., Aldering, G., Goldhaber, G. et al., 1999 ApJ 517, 565

Pospieszalska-Surdej, A., Surdej, J., Detal, A., Jean, C., 2000 Proceedings of the Colloquium "Gravitational Lensing: Recent Progress and Future Goals", Boston 1999, editors T. Brainerd \& C.S. Kochanek, in press; astro-ph/9910452

Porcas, R.W., Booth, R.S., Browne, I.W.A. et al. 1981 Nature 289, 758

Press, W.H., Gunn, J.E., 1973 ApJ 185, 397

Press, W.H., Rybicki, G.B., Hewitt, J.N, 1992a ApJ 385, 404

Press, W.H., Rybicki, G.B., Hewitt, J.N, 1992b ApJ 385, 416

Quast, R., Helbig, P., 1999 A\&A 344, 721

Racine, R., 1992 ApJ 395, L65

Rao, A.P., Subrahmanyan, R.: 1988, Monthly Notices Roy. Astron. Soc. 231, 229, [RAO88.1] Ratnatunga, K.U., Ostrander,E.J., Griffiths, R,E., Myungshin Im, 1995 ApJ 453, L5, [RAT95.1]

Ratnatunga, K.U., Griffiths, R.E. Ostrander, E.J., 1999 AJ 117, 2010, [RAT99.1]

Refsdal, S., 1964 MNRAS 128, 307

Refsdal, S., Surdej J., 1994 Rep. Prog. Phys. 56, 117

Reimers, D., Wisotzki, L., 1997 The Messenger (ESO) 88, 14

Reimers, D., Koehler, T.,Wisotzki, L., 1996, A\&AS 115, 235

Riess, A.G., Filippenko, A.V., Challis, P., et al., 1998 AJ 116, 1009

Rowan-Robinson, M., Broadhurst, T., Oliver, S. J. et al., 1991 Nature 351, 719, [ROW91.1]

Scarpa, R., Urry, C.M., Falomo, R., 1999 ApJ 521, 134

Schechter, P.L., Bailyn, C.D., Barr, R. et al., 1997 ApJ 475, L85

Schechter, P.L., Gregg, M.D., Becker, R.H. et al., 1998 AJ 115, 1371, [SCH98.2]

Schild, R., 1990 AJ 100, 1771

Schild, R., Thomson, D.J., 1995 AJ 109, 1970

Schild, R., Thomson, D.J., 1997 AJ 113, 130

Schneider, P., 1984 A\&A 140, 119

Schneider, P., 1985, A\&A 143, 413

Schneider, P., 1987, A\&A 183, 189

Schneider, P., Ehlers, J., Falco, E.E., 1992 "Gravitational Lenses" A\&A Library, (editors M. Harwitt, R. Kippenhahn, V. Trimble, J.-P. Zahn), Springer-Verlag.

Schneider, P., 1993 A\&A 279,

Schmidt R., Wambsganss, J., 1998 A\&A 335, 379

Schutz, B.F., 1985 "A first course in general relativity", Cambridge University Press

Smette, A., Surdej, J., Shaver, P.A., et al., 1992 ApJ 389, 39

Smette, A., Robertson, J.G., Shaver, et al., 1995 A\&AS 113, 199

Smette, A., Claeskens, J.-F., Surdej, J., 1997 New Astr. 2, 53

Smith, R.J., Boyle, B.J., Croom, S. et al. 1999 AAO Newsletter 91, 12

Surdej, J., Magain, P., Swings, J.-P., Borgeest, U. et al., 1987 Nature 329, 695, [SUR87.1]

Surdej, J., Magain, P., Swings, J.-P., Borgeest, U. et al., 1988a, A\&A 198, 49

Surdej, J., Magain, P., Swings, J.-P., Remy, M., Borgeest, U. et al., 1988b Proc. first DAEC Workshop on "Large Scale Structures", p. 95 (éditeurs Balkowski, C., Gordon, S.).

Surdej, J., Arnaud, J., Borgeest, U. et al., 1989 The Messenger (ESO) 55, 8

Surdej, J., Claeskens, J.-F., Crampton, D. et al., 1993a AJ 105, 2064

Surdej, J., Remy, M., Smette, A. et al., 1993b Proc. of the 31st Liège International Astrophysical Colloquium "Gravitational lenses in the Universe", pp 153-160 (editors J. Surdej, D. Fraipont-Caro, E. Gosset, S. Refsdal, M. Remy), [SUR93]

Surdej, J., Soucail, G., 1993 Proc. of the 31st Liège International Astrophysical Colloquium "Gravitational lenses in the Universe", p205 (editors J. Surdej, D. Fraipont-Caro, E. Gosset, S. Refsdal, M. Remy). 
Surdej, J., Claeskens, J.-F., 1997 in the Proc. of the Marseille Workshop entitled "Science with Liquid Mirror Telescopes", April 14-15 1997 (editor M. Ferrari), in press, ftp://vela.astro.ulg.ac.be/preprints/glslmt.ps.gz

Surdej, J., Claeskens, J.-F., Remy, M., 1997 A\&A 327, L1

Sykes, C.M., Browne, I.W.A., Jackson, N.J. et al., 1998 MNRAS 301, 310, [SYK98.1]

Toft, S., Hjorth, J., Burud, I., 2000 A\&A, in press, astro-ph/0003098, [TOF00.1]

Tonry, J.L., 1998 AJ 115, 1, [TON98.1]

Tonry, J.L., Franx, M., 1999 ApJ 515, 512

Tonry, J.L., Kochanek, C.S., 1999 AJ 117, 2034, [TON99.2]

Tonry, J.L., Kochanek, C.S., 2000 AJ 119, 1078, [TON00.1]

Turner, E.L., 1980 ApJ 242, L135

Turner, E.L., Ostriker, J., Gott, J., 1984 ApJ 284, 1

Turner, E.L., 1990 ApJ 365, L43

Tyson, J.A., 1988 AJ 96, 1

Vanderriest, C., Schneider, J., Herpe, G. et al. 1989 A\&A 215, 1

Van Dokkum, P.G., Franx, M., 1995 AJ 110, 2027

Véron-Cetty, M.-P., Véron, P., 1987, A catalogue of Quasars and Active Nuclei, ESO Scientific Report No. 5

Véron-Cetty, M.-P., Véron, P., 1991, A catalogue of Quasars and Active Nuclei, ESO Scientific Report No. 10

Véron-Cetty, M.-P., Véron, P., 1993, A catalogue of Quasars and Active Nuclei, ESO Scientific Report No. 13

Wallington, S., Narayan, R., 1993 ApJ 403, 517

Walsh, D., Carswell, R.F., Weymann, R.J., 1979 Nature 279, 381, [WAL79.1]

Wambsganss, J., 1998 Living Reviews in Relativity 1, No.12 and astro-ph/9812021

Warren, S.J., Hewett, P.C., Lewis, G.F. et al., 1996 MNRAS 278, 139, [WAR96.1]

Warren, S.J., Lewis, G.F., Hewett, P.C. et al., 1999, A\&A 343, L35, [WAR99.1]

Weinberg, S., 1972, Gravitation and cosmology Wiley, New-York

Weymann, R, Latham, D., Angel, J. et al. 1980 Nature 285, 641, [WEY80.1]

Wiklind, T., Combes, F., 1998 ApJ 500, 129, [WIK98.1]

Williams, L.L.R., Irwin, M., 1998 MNRAS 298, 378

Wisotzki, L., Kölher, T., Kayser, R., Reimers, D., 1993 A\&A 278, L15, [WIS93.1]

Wisotzki, L., Köhler, T., Lopez, S., Reimers, D., 1996a A\&A 315, L408, [WIS96.1]

Wisotzki, L., Koehler, T., Groote, D., Reimers, D., 1996b A\&AS 115, 227

Wisotzki, L., Wucknitz, O., Lopez, S., Soerensen, A.N., 1998 A\&A 339, L73, [WIS98.1]

Wisotzki, L., Chriestlieb, N., Liu, M.C. et al., 1999 A\&A 348, L41, [WIS99.1]

Wisotzki, L., $2000 A \& A$ in press, astro-ph/9911315

Wolfe A.M., 1988 in "QSO Absorption Lines: Probing the Universe", eds. J.C. Blades, D.A. Turnshek and C.A. Norman, Cambridge University Press, p297

Wyithe, R. L. Webster, E. L. Turner, E.L., Mortlock, D.J., 2000 MNRAS, submitted, astro$\mathrm{ph} / 9904361$

Xanthopoulos, E., Browne, I.W.A., King et al., 1998 MNRAS 300, 649, [XAN98.1]

Yee, H.K.C., 1988 AJ 95, 1331

Yee, H.K.C., Filippenko, A.V., Tang, D., 1993 AJ 105, 7

Yun, M.S., Scoville, N.Z., Carrasco, J.J., Blandford, R.D., 1997 ApJ 479, L9

Zwicky, F., 1937 Phys. Rev. Let. 51, 290

This article was processed by the author using the $\mathrm{IAT}_{\mathrm{E}} \mathrm{X}$ style file cljour 1 from Springer-Verlag. 